\title{
MAGNETIC FIELDS IN A SAMPLE OF NEARBY SPIRAL GALAXIES
}

\author{
C. L. Van Eck ${ }^{1}$, J. C. Brown ${ }^{2}$, A. Shukurov ${ }^{3}$, and A. Fletcher ${ }^{3}$ \\ ${ }^{1}$ Department of Astrophysics, Faculty of Science, Radboud University Nijmegen, P.O. Box 9010, 6500 GL Nijmegen, The Netherlands; c.vaneck@astro.ru.nl \\ ${ }^{2}$ Department of Physics and Astronomy, University of Calgary, Calgary, AB T2N 1N4, Canada; jocat@ ucalgary.ca \\ ${ }^{3}$ School of Mathematics and Statistics, Newcastle University, Newcastle upon Tyne NE1 7RU, UK; anvar.shukurov@ncl.ac.uk, andrew.fletcher@ncl.ac.uk \\ Received 2014 February 3; accepted 2014 November 5; published 2015 January 14
}

\begin{abstract}
Both observations and modeling of magnetic fields in the diffuse interstellar gas of spiral galaxies are well developed, but the theory has been confronted with observations for only a handful of individual galaxies. There is now sufficient data to consider the statistical properties of galactic magnetic fields. We have collected data from the literature on the magnetic fields and interstellar media of 20 spiral galaxies, and tested for various physically motivated correlations between magnetic field and interstellar medium parameters. Clear correlations emerge between the total magnetic field strength and molecular gas density as well as the star formation rate. The magnetic pitch angle exhibits correlations with the total gas density, the star formation rate, and the strength of the axisymmetric component of the mean magnetic field. The total and mean magnetic field strengths exhibit a noticeable degree of correlation, suggesting a universal behavior of the degree of order in galactic magnetic fields. We also compare the predictions of galactic dynamo theory to observed magnetic field parameters and identify directions in which theory and observations might be usefully developed.
\end{abstract}

Key words: galaxies: ISM - galaxies: magnetic fields - galaxies: spiral - magnetic fields magnetohydrodynamics (MHD) - radio continuum: ISM

\section{INTRODUCTION}

Magnetic fields are recognized as an essential component of the interstellar medium (ISM) in spiral galaxies. In particular, they confine cosmic rays (Berezinskii et al. 1990), contribute to disk-halo interactions (Norman \& Ikeuchi 1989; Kahn \& Brett 1993), transfer angular momentum in gas clouds to allow stars to form (e.g., Zweibel \& Heiles 1997), and provide vertical support of the interstellar gas (Boulares \& Cox 1990; Fletcher \& Shukurov 2001). The origin of galactic magnetic fields is plausibly connected to dynamo action (Beck et al. 1996; Shukurov 2007; see, however, Kulsrud 1999), but the details of their structure and evolution remain insufficiently explored and understood, either theoretically or observationally.

Our goal in this paper is to develop approaches to compare theory and observations of galactic magnetic fields to complement detailed studies of individual galaxies (Beck 2012) with an exploration of galaxy samples using statistical tools. As a first step in such an exploration, one has to identify specific combinations of observable galactic parameters that control magnetic fields in the framework of each theory.

The number of galaxies with well-explored magnetic fields has increased in recent years to a few dozen (e.g., Beck 2007; Chyży 2008; Fletcher et al. 2011). It is now possible to begin exploring galactic magnetic fields and their interconnections with other elements of the interstellar environment on a statistical level. This has been done for individual galaxies (e.g., Chyży 2008; Tabatabaei et al. 2013a, 2013b) and for a sample of dwarf irregular (Chyży et al. 2011) and normal spiral galaxies (Heesen et al. 2014), with the main emphasis on the radio-(far-)infrared and radio-star formation correlations, but without any deep comparison with theoretical models of galactic magnetic fields. Comparisons of the predictions of dynamo theory with observations were restricted to individual galaxies (Ruzmaikin \& Shukurov 1981; Ruzmaikin et al. 1985; Baryshnikova et al. 1987; Krasheninnikova et al. 1989; Starchenko \& Shukurov 1989; Moss et al. 1998; Rohde et al.
1999; Moss et al. 2001, 2007). It is compelling and imperative to clarify how statistical properties of magnetic fields in a sample of galaxies available compare with theoretical predictions. Apart from other outcomes, such an analysis would be able to suggest the most efficient directions for both observational and theoretical developments.

We present the data set used in Section 2, and identify galactic parameters and their combinations relevant to interstellar magnetic fields in Section 3. Comparison of the observational magnetic field parameters with predictions of the mean-field dynamo theory can be found in Section 4 and their relation to basic ISM parameters in Section 5. Our results are put into a broader perspective in Section 6 and summarized in Section 7. As part of our effort to keep the main text brief, we present additional details in the appendices.

\section{DATA}

We surveyed the literature to collect relevant information for a sample of nearby spiral galaxies; our survey resulted in data for 20 galaxies. A list of these galaxies with some noteworthy parameters is included in Table 1. In order to be considered for this study, the galaxy had to have a magnetic field strength (or at least its average value) reported for some clearly defined region (either by stating the radial range, or a qualitative description of the area observed). Then, we isolated the following parameters where they were available: strengths for the total and mean (large-scale) magnetic fields, $B$ and $\bar{B}$, respectively, the strength of the axisymmetric component of the mean magnetic field $\bar{B}_{0}$, the pitch angle of the mean magnetic field, $p_{B}$ (also referred to as the magnetic pitch angle), the mass surface densities of atomic and molecular hydrogen, $\Sigma_{\mathrm{I}}$ and $\Sigma_{2}$, respectively, the surface density of star formation rate (SFR) $\Sigma_{*}$, and the rotation curve, from which we calculated the angular velocity $\Omega$ and rotational shear $S=r \mathrm{~d} \Omega / \mathrm{d} r$.

The available estimates of the large-scale magnetic field are obtained from either the degree of polarization, assuming 
Table 1

The General Properties of the Sample Galaxies and the Method Used to Estimate the Total and Mean Magnetic Field Strengths, $B$ and $\bar{B}$, Given in Table 2

\begin{tabular}{|c|c|c|c|c|c|c|c|}
\hline \multirow{2}{*}{$\begin{array}{l}\text { Galaxy } \\
\text { (1) }\end{array}$} & \multicolumn{2}{|c|}{ Hubble Type $^{\mathrm{a}}$} & \multirow{2}{*}{$\begin{array}{c}\text { Distance }^{\mathrm{b}} \\
(\mathrm{Mpc}) \\
(4)\end{array}$} & \multirow{2}{*}{$\begin{array}{l}\text { Linear resolution } \\
\qquad(\mathrm{kpc}) \\
(5)\end{array}$} & \multirow{2}{*}{$\begin{array}{c}\text { Method }^{\mathrm{d}} \\
\text { (6) }\end{array}$} & \multicolumn{2}{|c|}{ Inclination $^{\mathrm{e}}\left({ }^{\circ}\right)$} \\
\hline & $\begin{array}{l}\text { NED } \\
(2)\end{array}$ & $\begin{array}{l}\text { LEDA } \\
\text { (3) }\end{array}$ & & & & $\begin{array}{c}\text { REF } \\
(7)\end{array}$ & $\begin{array}{c}\text { LEDA } \\
(8)\end{array}$ \\
\hline M31 & $\mathrm{SA}(\mathrm{s}) \mathrm{b}$ & $\mathrm{Sb}$ & 0.69 & $0.6 / 1.0 / 0.15$ & $\mathrm{E}$ & 78 & 72 \\
\hline M33 & $\mathrm{SA}(\mathrm{s}) \mathrm{cd}$ & $\mathrm{Sc}$ & 0.84 & 0.7 & $\mathrm{~F}$ & 56 & 55 \\
\hline M51 & $\mathrm{Sa}+\mathrm{Sc}$ & Sbc & 7.6 & 0.6 & $\mathrm{~F}$ & 20 & 33 \\
\hline M66 & $\mathrm{SAB}(\mathrm{s}) \mathrm{b}$ & $\mathrm{SABb}$ & 11.9 & 0.8 & $\mathrm{E}$ & $\ldots$ & 68 \\
\hline M81 & $\mathrm{SA}(\mathrm{s}) \mathrm{ab}$ & $\mathrm{Sab}$ & 3.25 & $0.7: 1.1$ & $\mathrm{E}$ & 59 & 63 \\
\hline M82 & I0 & Scd & 5.0 & $0.58: 0.17$ & $\mathrm{E}$ & $\ldots$ & 77 \\
\hline M94 & (R)SA(r)ab & $\mathrm{Sab}$ & 4.7 & 0.3 & $\mathrm{E}$ & 35 & 32 \\
\hline M99 & $\mathrm{SA}(\mathrm{s}) \mathrm{c}$ & $\mathrm{Sc}$ & 20.0 & 1.5 & $\mathrm{E}$ & 42 & 20 \\
\hline M104 & $\mathrm{SA}(\mathrm{s}) \mathrm{a}$ & $\mathrm{Sa}$ & 8.9 & 3.6 & $\mathrm{E}$ & 84 & 59 \\
\hline M109 & $\mathrm{SB}(\mathrm{rs}) \mathrm{bc}$ & Sbc & 15.0 & 2.2 & $\mathrm{E}$ & 59 & 47 \\
\hline NGC 253 & $\mathrm{SAB}(\mathrm{s}) \mathrm{c}$ & $\mathrm{SABc}$ & 3.94 & $0.6 / 1.6 / 2.8$ & $\mathrm{E}$ & 79 & 90 \\
\hline NGC 891 & $\mathrm{SA}(\mathrm{s}) \mathrm{b} ?$ & $\mathrm{Sb}$ & 7.2 & $1.4 / 2.8: 2.0$ & $\mathrm{E}$ & $\geqslant 88$ & 90 \\
\hline NGC 1365 & $\mathrm{SB}(\mathrm{s}) \mathrm{b}$ & $\mathrm{Sb}$ & 18.6 & 2.3 & $\mathrm{E}$ & 40 & 63 \\
\hline NGC 1566 & $\mathrm{SAB}(\mathrm{s}) \mathrm{bc}$ & $\mathrm{SABb}$ & 17.4 & 2.2 & $\mathrm{E}$ & 27 & 48 \\
\hline NGC 4414 & $\mathrm{SA}(\mathrm{rs}) \mathrm{c} ?$ & $\mathrm{Sc}$ & 19.2 & 1.5 & $\mathrm{E}$ & 55 & 57 \\
\hline NGC 5775 & $\mathrm{SBc} ?$ & $\mathrm{SBc}$ & 26.7 & 2.1 & $\mathrm{E}$ & 86 & 83 \\
\hline NGC 5907 & $\mathrm{SA}(\mathrm{s}) \mathrm{c} ?$ & $\mathrm{SABc}$ & 11.0 & 2.2 & $\mathrm{E}$ & 87 & 90 \\
\hline NGC 6946 & SAB(rs)cd & $\mathrm{SABc}$ & 5.5 & 0.4 & $\mathrm{E}$ & 38 & 18 \\
\hline IC 342 & SAB(rs)cd & $\mathrm{SABc}$ & 3.1 & $4 / 2.4$ & $\mathrm{E}$ & 25 & 19 \\
\hline
\end{tabular}

Notes. Blank entries $(.$.$) indicate that the data are not available. References can be found in Table 3$.

${ }^{a}$ Hubble type according to the NED (http://ned.ipac.caltech.edu) and LEDA (http://leda.univ-lyon1.fr) databases.

b Distance to the galaxy, mostly adopted to be same as in the original publication of the magnetic field data or, if not given there, of the rotation curve data (see Table 3).

c The linear resolution of the magnetic field observations: entries separated by a solidus are those at the individual observation wavelengths wherever the resolution of the data analysis is not specified; those separated by a colon represent the major and minor axes of the beam.

d The method used to estimate $B$ and $\bar{B}$ : equipartition with cosmic rays and the degree of polarization (E) or the Faraday rotation (F).

e The inclination angle, with $90^{\circ}$ corresponding to the edge-on view: entries taken from the sources shown in Table 3 are in Column (7), and those form the LEDA database, in Column (8).

energy equipartition with cosmic rays and most often neglecting any depolarization effects, and/or from the Faraday rotation, by measuring the rotation measure from polarization angles at two or more different frequencies. The former approach cannot distinguish between the genuine large-scale magnetic field and an anisotropic random magnetic field (summarily described as an ordered magnetic field), whereas the latter yields the true large-scale magnetic field weighted with thermal electron density. It is understandable then that the equipartition estimates are systematically higher than those from Faraday rotation. Stepanov et al. (2014) show, with M33 as an example, that the difference is consistent with the expected degree of anisotropy of the random magnetic fields (see also Iacobelli et al. 2013). On the other hand, magnetic field estimates from Faraday rotation depend on the assumption of the correlation between magnetic field strength and thermal electron density; this plausibly introduces systematic bias into magnetic fields strengths obtained (Beck et al. 2003).

Fourteen galaxies in the sample have the average magnetic field strength published, but not the precise averaging region. Therefore, we had to make some assumptions about the averaging regions used. We chose to use the extent of polarized emission along the major axis of the galaxy as the diameter over which to average; we defined the extent of polarized emission as the location of the lowest contour of the polarized intensity map that is always included in such publications. Galaxies falling into this category are listed in Table 2 with asterisks.

For some of the nearest galaxies (e.g., M31, M33, M51, and M81), the strength of the mean magnetic field is reported for a series of concentric rings of well-defined radii. These estimates are more reliable as they are obtained from the differences between the polarization angles observed at several wavelengths and allow for depolarization effects if necessary. In such cases, the averaging region for the magnetic field is well defined, and it was possible to ensure that the values used for the other parameters cover the same region of the galaxy. For NGC 6946, the energy density of the azimuthally averaged total and mean magnetic fields were published in graphical form as continuous functions of the galactocentric radius. For IC 342, an average magnetic field within the galactocentric radius $13.5 \mathrm{kpc}$ is known. These data are shown in Table 2 without the asterisk on their radial range. We also used the data for individual rings wherever available.

For five of the galaxies in the sample (M31, M33, M51, NGC 1097, and NGC 1365) detailed modeling had been used to separate the different azimuthal components of the largescale magnetic field in different radial ranges (i.e., identifying the amplitudes $\bar{B}_{m}$ of the azimuthal Fourier modes given by $\bar{B}=\sum_{m} \bar{B}_{m} \cos m\left(\theta-\beta_{m}\right)$, where $\theta$ is the azimuthal position in the galaxy disk and $\beta_{m}$ the phase of the mode). For these galaxies we have also used the strength of the axisymmetric $m=0$ mode $\bar{B}_{0}$ as this is expected to have the fastest growth rate and cover the largest radial range according to the standard mean-field galactic dynamo theory (Ruzmaikin et al. 1988b).

The linear resolution of radio observations, shown in Table 1, often exceeds the scale of the mean magnetic field, which is of the order of $1 \mathrm{kpc}$. The data can be corrected for unresolved 
Table 2

The Magnetic Field and ISM Parameters, in the Radial Ranges Specified

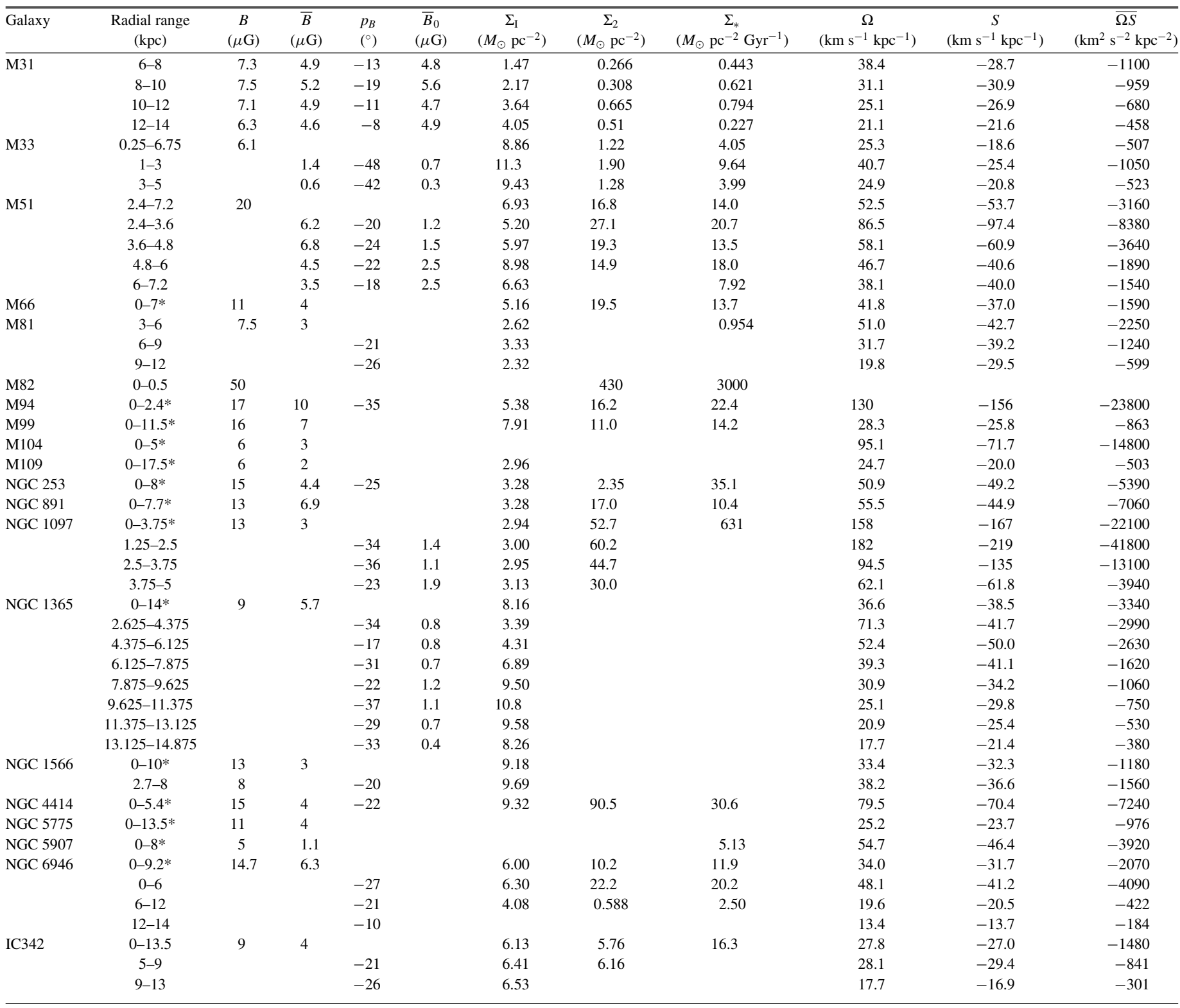

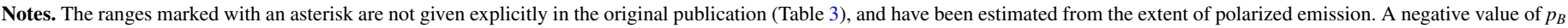

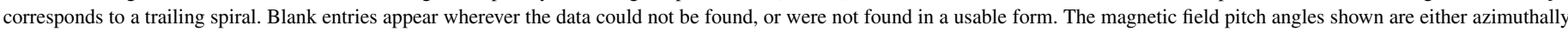

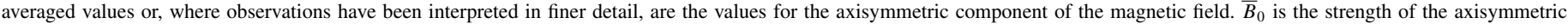
component of the mean magnetic field.

gradients of magnetic field as described in Appendix A, but we found this correction insignificant in our sample.

Only a small fraction of the galaxies in our sample also have magnetic pitch angles reported, most often as averages over specific radial ranges. For three galaxies (M94, NGC 253, and NGC 4414) the average pitch angle is given without specifying the averaging region. For these galaxies, we defined the region of averaging as the extent of polarization, as we did for the field strengths. The magnetic pitch angle values are given in Table 2.

For other relevant parameters of galaxies, we computed the area averages by integrating each quantity of interest over the same radial range where the magnetic field was averaged, as given in Table 2. Since the magnetic field strength and pitch angle data were averaged over different radial ranges in some cases, we computed the averages separately for each such radial range. The average value of a product of variables can be rather different from the product of their averages; the mean values shown and used in such cases were obtained by averaging the corresponding products. The sources of the data are given in Table 3.

The data we collected are summarized in Figure 1, which shows some parameters normalized to their mean value in the sample. This figure illustrates the properties of our galaxy sample. The magnetic pitch angle has the narrowest distribution in the sample, $8^{\circ} \leqslant\left|p_{B}\right| \leqslant 37^{\circ}$. The magnetic field strength has a relatively narrow distribution, with a spread of one order of magnitude, $5 \lesssim B \lesssim 50 \mu \mathrm{G}$ and $1 \lesssim \bar{B} \lesssim 10 \mu \mathrm{G}$. However, a more physically relevant quantity, the magnetic energy density, has a spread of two orders of magnitude, comparable to that in the other parameters. For comparison, 


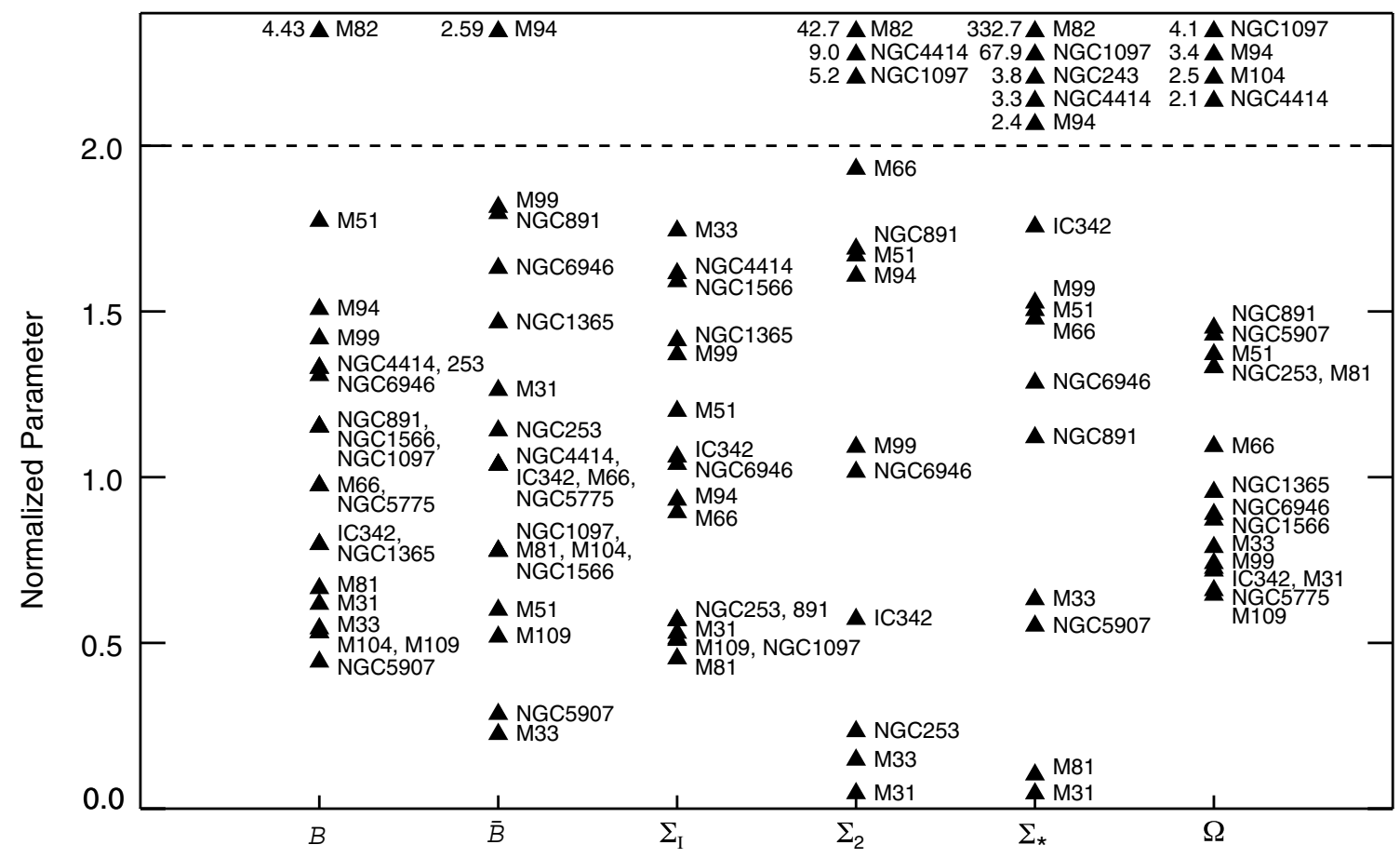

Figure 1. Visual summary of the galactic parameters in the sample, normalized to their mean values. From left to right: $B$-the total magnetic field; $\bar{B}$-the mean magnetic field ; $\Sigma_{\mathrm{I}}$-the surface density of neutral hydrogen $\left(\mathrm{HI}_{\mathrm{I}}\right) ; \Sigma_{2}$ — the surface density of molecular hydrogen $\left(H_{2}\right) ; \Sigma_{*}$-SFR per unit area; $\Omega$-angular velocity. Average values for each parameter were calculated using galaxies below the dashed line. As an example, the total magnetic field for M82 has 4.43 times the average total magnetic field for the remaining galaxies in the column (all below the dashed line), while M66 is roughly at the average (as indicated by its position close to 1.0 normalized parameter). From Table $2, B$ for M82 is $50 \mu \mathrm{G}=4.43 \times 11.29 \mu \mathrm{G}$, while $B$ for M66 is $11 \mu \mathrm{G}$. This method of normalization was done solely to avoid crowding in the plot and allows individual galaxies to be identified in the distribution of the parameters.

Table 3

Data Sources

\begin{tabular}{|c|c|c|c|c|}
\hline Galaxy & $B, \bar{B}, \bar{B}_{0}$ and Inclination & Rotation Curve & Gas Density & Star Formation Rate \\
\hline M31 (NGC 224) & Fletcher et al. (2004) & Sofue et al. (1999) & Boissier et al. (2007) & Tabatabaei \& Berkhuijsen (2010) \\
\hline M33 (NGC 598) & Tabatabaei et al. (2008) & Sofue et al. (1999) & Boissier et al. (2007) & Verley et al. (2009) \\
\hline M51 (NGC 5194) & Fletcher et al. (2011) & Sofue et al. (1999) & Leroy et al. (2008) & Leroy et al. (2008) \\
\hline M66 (NGC 3627) & Soida et al. (2001) & de Blok et al. (2008) & Leroy et al. (2008) & Leroy et al. (2008) \\
\hline M81 (NGC 3031) & Krause et al. (1989) & Sofue et al. (1999) & Boissier et al. (2007) & Calzetti et al. (2010) \\
\hline M82 (NGC 3034) & Klein et al. (1988) & Sofue et al. (1999) & Lo et al. (1987) & Lo et al. (1987) \\
\hline M94 (NGC 4736) & Chyży \& Buta (2008) & de Blok et al. (2008) & Crosthwaite (2001) & Leroy et al. (2008) \\
\hline M99 (NGC 4254) & Chyży (2008) & Dicaire et al. (2008) & Warmels (1988) & Rahman et al. (2011) \\
\hline M104 (NGC 4594) & Krause et al. (2006) & Tempel \& Tenjes (2006) & $\cdots$ & $\cdots$ \\
\hline M109 (NGC 3992) & Beck et al. (2002) & Bottema \& Verheijen (2002) & Bottema \& Verheijen (2002) & $\cdots$ \\
\hline NGC 253 & Heesen et al. (2009) & Sofue et al. (1999) & Sorai et al. (2000) & Waller et al. (1988) \\
\hline NGC 891 & Hummel et al. (1991) & Yim et al. (2011) & Yim et al. (2011) & Yim et al. (2011) \\
\hline NGC 1097 & Beck et al. (2005) & Sofue et al. (1999) & Crosthwaite (2001) & Kennicutt (1998) \\
\hline NGC 1365 & Beck et al. (2005) & Sofue et al. (1999) & Jałocha et al. (2010) & $\cdots$ \\
\hline NGC 1566 & Ehle et al. (1996) & Sofue et al. (1999) & Becker et al. (1988) & $\cdots$ \\
\hline NGC 4414 & Soida et al. (2002) & Fridman et al. (2005) & Thornley \& Mundy (1997) & Wong \& Blitz (2002) \\
\hline NGC 5775 & Soida et al. (2011) & Heald et al. (2006) & $\cdots$ & $\cdots$ \\
\hline NGC 5907 & Dumke et al. (2000) & Brownstein \& Moffat (2006) & $\cdots$ & Misiriotis et al. (2001) \\
\hline NGC 6946 & Beck (2007); Ehle \& Beck (1993) & de Blok et al. (2008) & Leroy et al. (2008) & Leroy et al. (2008) \\
\hline IC 342 & Gräve \& Beck (1988) & Sofue et al. (1999) & Crosthwaite (2001) & Calzetti et al. (2010) \\
\hline
\end{tabular}

the mass surface densities of molecular hydrogen and the SFR also span two orders of magnitude, $0.5 \lesssim \Sigma_{2} \lesssim 100 M_{\odot} \mathrm{pc}^{-2}$ and $0.4 \lesssim \Sigma_{*} \lesssim 30 M_{\odot} \mathrm{pc}^{-2} \mathrm{Gyr}^{-1}$ (excluding M82 that has an extremely high surface density of SFR as well as other parameters because the data refer to the central part of the galaxy). Altogether, the sample contains 20 galaxies in almost all the variables required for analysis based on the concepts presented below.

Before verifying the theoretical predictions discussed in Section 3, we perform a consistency check of the data collected, and their averaging, by testing the Schmidt-Kennicutt law (Kennicutt 1989), an empirical relation between the surface mass density of the interstellar gas $\Sigma=\Sigma_{\mathrm{I}}+\Sigma_{2}$ and that of star formation $\Sigma_{*}$. Indeed, the data are consistent with

$$
\Sigma_{*} \propto \Sigma^{1.25 \pm 0.41}
$$

with a Spearman correlation coefficient of 0.52. This agrees fairly well with Kennicutt (1989), who found a power-law index of $1.3 \pm 0.3$, with Spearman correlation coefficients between 0.49 and 0.76 . 
We cannot be similarly encouraged by the quality of the data on the galactic magnetic fields. Most of the estimates of both the total and mean magnetic field strengths are determined from energy equipartition with cosmic rays, often using uncertain estimates of the path length and the relativistic proton-electron ratio, imperfect (if any) separation of thermal and nonthermal contributions to the total radio intensity, etc. Furthermore, the size of the averaging region used in the estimates varies vastly between the galaxies, and it is not clear in advance if such data can be used to test any theory at all. Therefore, we first focus on a few nearby galaxies where parameters of the mean magnetic field were obtained from well-defined fits of azimuthal Fourier modes to multi-frequency observations of the polarization angles within relatively narrow rings that pass statistical goodness-of-fit tests. These are the best data on the galactic mean magnetic fields available. Our discussion of the magnetic fields obtained from equipartition arguments is only exploratory and serves mostly to assess their quality and clarify the most important improvements required in the interpretation of the radio astronomical data.

\section{ASTROPHYSICAL CONTEXT FOR THE DATA INTERPRETATION}

Compression and stretching are the two fundamental processes that can affect the magnetic field, $\boldsymbol{B}$. In a random flow, they can result in the generation of a self-sustained magnetic field via various forms of dynamo action, both at "large" scales (larger than the correlation scale of the random flow) to produce a mean (large-scale) magnetic field $\overline{\boldsymbol{B}}$, and at the range of scales of the random motions themselves (the "small" scales) to generate a random magnetic field $\boldsymbol{b}$ in a wide range of scales. Interstellar turbulence is transonic, so that both stretching and compression affect interstellar magnetic fields. In this section we briefly discuss the most important processes thought to affect interstellar magnetic fields and their dependence on galactic parameters.

\subsection{Compression and Stretching of Magnetic Fields}

In relatively simple flows, connection between gas density and velocity resulting from the continuity equation can be tractable, and magnetic field strength can be expressed as a function of gas density $\rho$ alone. For example, magnetic flux through any moving contour is conserved in an ideal plasma. Together with mass conservation, this results in a power-law dependence of magnetic field strength on gas density $\rho$,

$$
B \propto \rho^{k}
$$

with $k=2 / 3$ for a spherically symmetric collapse, $k=1 / 2$ for an anisotropic compression into a flattened cloud via a sequence of quasi-equilibrium states (Mestel \& Paris 1984), and $k=1$ for a one-dimensional compression, as in a shock.

Correlations consistent with Equation (1), with $k \approx 0.65$ in denser clouds with particle number density $n$ in excess of $300 \mathrm{~cm}^{-3}$ (Calzetti et al. 2010), are well known from Zeeman measurements (Troland \& Heiles 1986). However, Basu (2000) noticed that magnetic field strength in dense interstellar clouds has a tighter correlation with kinetic energy density within the cloud,

$$
B^{2} \propto \rho v^{2}
$$

rather than the gas density alone. Scaling of magnetic field strength with kinetic energy density is a typical feature of dynamo mechanisms, a kinetic-to-magnetic energy conversion.
For our study, the physically distinct correlations as presented in Equations (1) and (2) cannot be distinguished from each other. The intra-cloud velocity dispersions are not available for external galaxies as the resolution of either $\mathrm{CO}$ or $\mathrm{HI}$ observations is not sufficient to separate the intra-cloud velocity dispersion from that arising from the relative random motions of individual clouds.

Compression and stretching at large scales introduce anisotropy to otherwise isotropic random magnetic fields (Beck et al. 2005). Synchrotron emission from an anisotropic random magnetic field can be polarized (Sokoloff et al. 1998), making it more difficult to interpret the polarization of galactic radio emission in terms of large-scale magnetic fields. The anisotropy expected from stretching by the galactic differential rotation can account for a degree of polarization of the order of $10 \%$ (Section 2.1 in Stepanov et al. 2014). Calculations of anisotropic random magnetic fields produced by both galactic differential rotation and large-scale shocks can be found in Beck et al. (2005).

Compression by spiral arms and stretching by the associated streaming motions affect systematically the orientation of magnetic field at both large and small scales. In particular, magnetic field lines are refracted to a better alignment with the spiral arms within them. If the angle between the arm axis and magnetic field is $p_{1}$ between the arms and $p_{2}$ within them, one-dimensional compression leads to

$$
\tan p_{2}=\frac{\rho_{1}}{\rho_{2}} \tan p_{1}<\tan p_{1}
$$

where $\rho_{1}$ and $\rho_{2}$ are the gas densities between the arms and within the arm, respectively. As an example, $p_{2} \approx 5^{\circ}$ for $p_{1}=20^{\circ}$ and $\rho_{2} / \rho_{1}=4$. Streaming motions can improve the alignment even further.

\subsection{Origin of Galactic Magnetic Fields}

Large-scale (mean) magnetic fields coherent at a scale of the order of $1-10 \mathrm{kpc}$ are a common feature of spiral galaxies; they have been detected in all spiral galaxies observed with adequate sensitivity and resolution. Their widespread presence calls for a universal mechanism of their generation and maintenance. Two such mechanisms have been suggested: the turbulent mean-field dynamo theory (Ruzmaikin et al. 1988b) and the primordial field theory (Kulsrud 1999). Unlike the dynamo theory, the primordial concept does not lead to any specific predictions for the galactic magnetic fields, and its main unresolved problem is to explain consistently such basic parameters as the magnetic pitch angle and the predominantly quadrupolar parity of the large-scale magnetic fields (Shukurov 2007).

A primordial magnetic field may still serve as a seed magnetic field for galactic dynamos (Kulsrud \& Zweibel 2008), but otherwise this theory appears to be ruled out by the current state of observations. The cosmic dynamo theory does not require any hypothetical primordial magnetic field to launch the large-scale dynamo action. Instead, magnetic fields produced by battery mechanisms in stars, expelled into the ISM by stellar winds and supernova explosions, and then further amplified at a timescale of the order of $10^{7}-10^{8} \mathrm{yr}$ by the fluctuation dynamo in the interstellar medium (Shukurov 2007; Shukurov \& Sokoloff 2008) provide a seed magnetic field whose effective strength is of the order of $10^{-9} \mathrm{G}$ at a scale of a few kiloparsecs (Ruzmaikin et al. 1988a; Beck et al. 1994), which is quite sufficient to explain the observed large-scale magnetic fields in the framework of the mean-field dynamo theory. 
Galactic dynamo theory is well developed in the kinematic regime that describes the early stages of magnetic field growth, when the Lorentz force is still negligible in comparison with other forces in the ISM. However, the non-linear behavior of the interstellar magnetic field, when it approaches a statistically steady state, remains controversial, for both large- and smallscale dynamos.

In what follows, we present estimates of the steady-state strength of the large-scale magnetic field in spiral galaxies $\bar{B}$ that follow from the mean-field dynamo theory, with allowance for the uncertainties in its non-linear aspects. Throughout this paper, we use cylindrical polar coordinates $(r, \phi, z)$ with the origin at the galaxy's center and the $z$-axis aligned with the galactic angular velocity $\boldsymbol{\Omega}$.

\subsection{Basics of Galactic Dynamos}

Star formation in spiral galaxies, resulting in supernova explosions and galactic fountains and winds, drives ubiquitous transonic and supersonic random flows at scales of the order of $l \simeq 100 \mathrm{pc}$ and less. Together with the velocity field, interstellar magnetic fields can be naturally and usefully represented as a sum of two physically distinct parts, a mean field $\bar{B}$ (at scales of the order of $1 \mathrm{kpc}$ and more) and a random field $\boldsymbol{b}$ (at scales of $100 \mathrm{pc}$ and less). The mean and random magnetic fields are produced by different (albeit related) physical mechanisms. As we discuss below, the former are produced by the meanfield dynamo action that relies on density stratification of the galactic disks and their differential rotation. At kiloparsec scales, magnetic fields are further modified by the spiral pattern and galactic outflows.

Like other constituents of the ISM, the large-scale magnetic field is strongly affected by the multi-phase gas structure, and different phases play different roles in its generation and evolution (Shukurov 2007). Dense, cold clouds occupy a negligible fraction of the total volume, and field lines in the densest molecular clouds are subjected to enhanced magnetic reconnection, so this phase is not likely to host the large-scale dynamo. The hot gas is buoyant and leaves the disk for the galactic halo on a timescale shorter than the mean-field dynamo amplification time. The only pervasive, diffuse phase left as a candidate to host the mean-field dynamo action is the warm gas, which remains in a well-defined layer (despite being partially entrained in galactic outflows), and is partially ionized. The warm gas occupies a significant fraction of the volume, hence probably forms a connected region, and would thereby be able to accommodate the magnetic field coherent over kiloparsec scales. Thus, the warm phase has all the properties required to be the site of the mean-field dynamo action. Its parameters will be used in our discussion of the large-scale magnetic fields.

Random magnetic fields can be produced by another dynamo mechanism, the fluctuation dynamo, which acts, to a large extent, independently of the amplification of the large-scale magnetic field. This mechanism does not require anything more than a random plasma flow of sufficient intensity. Random magnetic fields are also produced by tangling of the mean magnetic field (in fact, this process is an essential part of the mean-field dynamo) and further modified by interstellar shocks. Hence, the mean and random magnetic fields are sensitive to distinct features of the plasma flow and thus depend on different galactic parameters. Therefore, they should be carefully separated before any meaningful relations to galactic parameters can be established.
The intensity of the induction effects producing a large-scale magnetic field, relative to its dissipation by ohmic resistivity, which is enhanced by the tangling of magnetic field lines by the random flow, can be quantified using the (dimensionless) turbulent magnetic Reynolds numbers

$$
R_{\alpha}=\frac{\alpha h}{\beta} \quad R_{\omega}=\frac{S h^{2}}{\beta}
$$

where

$$
\alpha \simeq l^{2} \Omega / h
$$

is a measure of the large-scale induction effects due to the helical random flows (arising from the systematic effects of the Coriolis force on the stratified galactic turbulence),

$$
\beta \simeq \frac{1}{3} l v
$$

is the turbulent magnetic diffusivity, $h$ is the pressure scale height, $l$ and $v$ are the turbulent scale length and velocity, and $S=r \mathrm{~d} \Omega / \mathrm{d} r$ is the large-scale velocity shear rate due to the galactic differential rotation. In the widely used approximation of an $\alpha \omega$-dynamo (where the induction effects of the galactic differential rotation are considered to be much stronger than the production of the large-scale magnetic field by galactic turbulence), it is useful to introduce the product of the two Reynolds numbers known as the dynamo number,

$$
D=R_{\alpha} R_{\omega} .
$$

The dimensionless dynamo control parameters $R_{\alpha}, R_{\omega}$, and $D$ vary with the galactocentric radius $r$, mainly because $\alpha, \Omega, S$, and $h$ depend on $r$. All the variables entering these definitions are observable, at least in principle:

$$
\begin{aligned}
R_{\alpha} \simeq & 3 \frac{l \Omega}{v}=0.75\left(\frac{\Omega}{25 \mathrm{~km} \mathrm{~s}^{-1} \mathrm{kpc}^{-1}}\right) \\
& \times\left(\frac{l}{0.1 \mathrm{kpc}}\right)\left(\frac{v}{10 \mathrm{~km} \mathrm{~s}^{-1}}\right)^{-1}
\end{aligned}
$$

and

$$
\begin{aligned}
R_{\omega} \simeq & 3 \frac{S h^{2}}{l v}=19\left(\frac{S}{25 \mathrm{~km} \mathrm{~s}^{-1} \mathrm{kpc}^{-1}}\right)\left(\frac{h}{0.5 \mathrm{kpc}}\right)^{2} \\
& \times\left(\frac{l}{0.1 \mathrm{kpc}}\right)^{-1}\left(\frac{v}{10 \mathrm{~km} \mathrm{~s}^{-1}}\right)^{-1},
\end{aligned}
$$

where $h$ is the (pressure) scale height of the warm gas, as above, and $r$ is the galactocentric radius. Since $\Omega$ usually decreases with $r$, we have $S<0, D<0$, and $R_{\omega}<0$ in most cases.

Thus, the dynamo number becomes

$$
\begin{aligned}
D \simeq & 9 \frac{\Omega S h^{2}}{v^{2}}=14\left(\frac{\Omega S}{625 \mathrm{~km}^{2} \mathrm{~s}^{-2} \mathrm{kpc}^{-2}}\right) \\
& \times\left(\frac{h}{0.5 \mathrm{kpc}}\right)^{2}\left(\frac{v}{10 \mathrm{~km} \mathrm{~s}^{-1}}\right)^{-2} .
\end{aligned}
$$

In most (if not all) spiral galaxies, the mean-field dynamos generate a basic axisymmetric mean magnetic field which is further modified by the spiral pattern, bar, etc., to add non-axisymmetric components (Beck et al. 1996). Therefore, predictions of the dynamo theory should be compared not 
with the total mean magnetic field observed, but with its axisymmetric part. We have done this whenever possible (see Table 2), but most observational results do not provide this information, limiting the number of galaxies with which we could compare.

\subsection{Mean Magnetic Field Strength}

The mean-field dynamo theory offers a range of estimates of the steady-state strength of the mean magnetic field whose complexity reflects the amount of detail in the underlying theory. We present here three such estimates: first a generic case, which only relies on the fundamental aspects of dynamo action, and then two further, more involved, models.

\subsubsection{Equipartition of Magnetic and Turbulent Energies}

The simplest estimate of magnetic field strength in the steady state relies on the fact that magnetic field energy is obtained from the kinetic energy of interstellar turbulence, so the statistically steady state can be expected to have comparable magnetic and turbulent kinetic energies, $\bar{B}^{2}=4 \pi \xi \rho v^{2}$, where $\rho$ is the gas density, $v$ is the rms random velocity, and $\xi$ is a factor of the order of unity. Later refinements of this estimate in Sections 3.4.2 and 3.4.3 represent, in fact, a clarification of the dependence of $\xi$ on physical parameters.

Under the simplest bifurcation (which takes place in the mean-field dynamo in a thin galactic disk), the energy density of the steady-state large-scale magnetic field will also be proportional to the deviation of the dynamo control parameter (i.e., the dynamo number) $D$ from its marginal (critical) value $D_{\text {cr }}$ with respect to the dynamo action (such that the large-scale magnetic field grows if $|D|>\left|D_{\mathrm{cr}}\right|$ and decays otherwise):

$$
\bar{B}^{2} \simeq 4 \pi \rho v^{2}\left(\frac{D}{D_{\mathrm{cr}}}-1\right) \text { for } \frac{D}{D_{\mathrm{cr}}}>1 \quad \bar{B}=0 \text { otherwise }
$$

where $\rho$ is the density of the warm gas and we use $\xi=1$.

Equation (11) can be written in terms of the surface density of the warm gas, approximated by that of neutral hydrogen $\Sigma_{\mathrm{I}}=2 h \rho$ :

$$
\begin{aligned}
\bar{B}^{2}= & (0.8 \mu \mathrm{G})^{2}\left(\frac{D}{D_{\mathrm{cr}}}-1\right)\left(\frac{\Sigma_{\mathrm{I}}}{1 M_{\odot} \mathrm{pc}^{-2}}\right)\left(\frac{h}{0.5 \mathrm{kpc}}\right)^{-1} \\
& \times\left(\frac{v}{10 \mathrm{~km} \mathrm{~s}^{-1}}\right)^{2}
\end{aligned}
$$

for $D / D_{\mathrm{cr}}>1$. This expression establishes the relation between the strength of the mean magnetic field and other directly observable galactic parameters resulting from the general concept of equipartition between magnetic and turbulent energy densities. It will be tested for the sample galaxies in Sections 4 and 5 .

In the simplest dynamo models, $D_{\mathrm{cr}}$ is a constant depending on the specific form of $\alpha$ as a function of $z$, of which we only know that $\alpha$ is an odd function of $z$ and presumably varies with $r$ as given in Equation (5). For a quadrupolar magnetic field, predominant in a thin-disk dynamo, the critical dynamo number remains within a relatively narrow range, $-4 \leqslant D_{\text {cr }} \leqslant-13$, for very broad and diverse range of the model forms of $\alpha(z)$ (Ruzmaikin et al. 1988b). When using such simple dynamo models, we select $D_{\text {cr }}=-8$ as a suitable estimate near the middle of this range; this corresponds to $\alpha \propto \sin (\pi z / h)$.
We discuss in Section 3.4.3, however, that $D_{\text {cr }}$ may depend on the speed of the galactic outflow (fountain or wind); then Equation (16) is appropriate.

\subsubsection{Magnetostrophic Balance}

A more physically detailed estimate of the steady-state mean magnetic field can be obtained by considering more carefully the mechanism by which the dynamo may saturate. The generation of the large-scale magnetic field relies on the mean helicity of interstellar random flows; the mean helicity is due to density stratification and galactic rotation combining to twist rising or sinking turbulent cells via the azimuthal component of the Coriolis force $\mathcal{C}=2 \rho[\boldsymbol{v} \times \boldsymbol{\Omega}]_{\phi} \simeq 2 \rho v_{r} \Omega$, written in the local cylindrical frame centered at the expanding turbulent cell. With the $z$-axis aligned with the galactic angular velocity $\boldsymbol{\Omega}$, the radial (expansion) velocity $v_{r}$ within the cell follows from the mass conservation $\nabla \cdot \boldsymbol{v}=0$ as $v_{r} \simeq v_{z} l / h$ in terms of the vertical component $v_{z}$. The azimuthal component of the Lorentz force produced by the large-scale magnetic field perturbed at the turbulent scale $l$ is given by $\mathcal{L}=(4 \pi)^{-1}[(\nabla \times \boldsymbol{B}) \times$ $\boldsymbol{B}]_{\phi} \simeq B_{r} B_{\phi} /(4 \pi l)$. The steady-state strength of the large-scale magnetic field then follows from the balance of the Coriolis and Lorentz forces, $\mathcal{C}+\mathcal{L} \simeq 0$ (the magnetostrophic balance), as (see also Ruzmaikin et al. 1988a)

$$
\bar{B}_{r} \bar{B}_{\phi} \simeq-\frac{8 \pi}{\sqrt{3}} \rho v \alpha
$$

where we have used Equation (5) and assumed isotropy of the interstellar turbulence, $v_{z}=v / \sqrt{3}$. This estimate relies on the plausible assumption that the dynamo action settles to a steady state because the Lorentz force affects the turbulent flow by opposing the Coriolis force that makes the flow helical, i.e., the back-reaction of magnetic field on the flow affects primarily the flow helicity $\left(R_{\alpha}\right)$ rather than the differential rotation $\left(R_{\omega}\right)$, which is supported by the stronger gravitational forces.

The radial and azimuthal components of the large-scale magnetic fields $\bar{B}$ are related via the pitch angle of magnetic lines,

$$
\bar{B}_{r}=\bar{B} \sin p_{B} \quad \bar{B}_{\phi}=\bar{B} \cos p_{B}
$$

and $p_{B}$ can be taken either from observations or from theory; various theoretical estimates of $\tan p_{B}$ can be found in Section 3.5.

In terms of observable parameters, and including the same factor with $D / D_{\text {cr }}-1$ as above, we obtain

$$
\begin{aligned}
\bar{B}^{2} \simeq & -\frac{16 \pi}{\sqrt{3}} \frac{\rho v \alpha}{\sin 2 p_{B}}\left(\frac{D}{D_{\mathrm{cr}}}-1\right) \\
= & -\frac{(0.3 \mu \mathrm{G})^{2}}{\sin 2 p_{B}}\left(\frac{D}{D_{\mathrm{cr}}}-1\right)\left(\frac{\Sigma_{\mathrm{I}}}{1 M_{\odot} \mathrm{pc}^{-2}}\right)\left(\frac{l}{0.1 \mathrm{kpc}}\right)^{2} \\
& \times\left(\frac{h}{0.5 \mathrm{kpc}}\right)^{-2}\left(\frac{v}{10 \mathrm{~km} \mathrm{~s}^{-1}}\right)\left(\frac{\Omega}{25 \mathrm{~km} \mathrm{~s}^{-1} \mathrm{kpc}^{-1}}\right)
\end{aligned}
$$

and note that $\bar{B}^{2}>0$ as long as $p_{B}<0\left(\right.$ and $\left.D / D_{\mathrm{cr}}>1\right)$.

\subsubsection{Magnetic Helicity Balance}

The mean-field dynamo action can be saturated not via the magnetostrophic balance, but rather because the dynamo action is suppressed by the build-up of magnetic helicity in the galactic disk before the balance is achieved. Shukurov et al. (2006) showed that galactic outflows (fountains or winds) 
can prevent a catastrophic quenching of the dynamo action by the accumulation of the magnetic helicity, and Sur et al. (2007) provide an estimate of the resulting steady-state mean magnetic field. Kleeorin et al. (2000) suggested that the turbulent diffusion of magnetic helicity can provide another way to remove it from the dynamo region (see also Kleeorin et al. 2002, 2003). Although the diffusive helicity transport still needs to be justified rigorously, we also include it in our estimates following Chamandy et al. (2014). There are other helicity fluxes that may contribute to the nonlinear state of the mean-field dynamo (Vishniac \& Cho 2001; Vishniac \& Shapovalov 2014), but they are less certain, depend on the anisotropy of the random flow, and are thus harder to quantify in terms of observable quantities. As we neglect them, it can be expected that the steadystate magnetic field strength is somewhat underestimated, and therefore we focus on its dependence on galactic parameters rather than its magnitude.

The resulting estimate of the mean magnetic field strength, which allows for both advective and diffusive fluxes of magnetic helicity, is based on a fully nonlinear theory, and thus the factor $D / D_{\text {cr }}-1$ emerges automatically. As shown by Chamandy et al. (2014) this estimate has the form

$$
\bar{B}^{2} \simeq\left(\frac{l}{h}\right)^{2} \frac{2 \pi \rho v^{2}\left(R_{U}+\pi^{2}\right)}{1-3 \sqrt{2} \cos ^{2}\left(p_{B}\right) / 8}\left(\frac{D}{D_{\mathrm{cr}}}-1\right)
$$

where $R_{U}$ is the turbulent Reynolds number for $U_{z}$, the massaveraged vertical velocity in the disk,

$$
R_{U}=\frac{U_{z} h}{\beta} \quad \text { with } \quad U_{z} \simeq f V_{z} \frac{n_{\mathrm{h}}}{n}
$$

$f$ is the fraction of the disk surface occupied by the outflow, mostly the hot gas, $V_{z}$ is the bulk vertical velocity of the hot gas, and $n_{\mathrm{h}} \simeq 10^{-3} \mathrm{~cm}^{-3}$ and $n \simeq 0.1 \mathrm{~cm}^{-3}$ are the gas number densities in the hot and warm phases, respectively, (assuming that the warm gas hosts the mean magnetic field, whereas the outflow mainly consists of the hot gas). With $f=0.1$, the fractional area of the disk surface occupied by the OB associations and chimneys (Norman \& Ikeuchi 1989) and $V_{z} \simeq 200 \mathrm{~km} \mathrm{~s}^{-1}$, typical of galactic fountains, this yields $U_{z} \simeq 0.2 \mathrm{~km} \mathrm{~s}^{-1}$.

The gas outflow also hinders the dynamo action, and thus affects the critical dynamo number that in fact depends on the outflow speed (Chamandy et al. 2014):

$$
D_{\mathrm{cr}} \simeq-\left(\frac{\pi}{2}\right)^{5}\left(1+\frac{R_{U}}{\pi^{2}}\right)^{2}
$$

so that $\left|D_{\text {cr }}\right|$ increases with $R_{U}$. The critical dynamo number obtained for $R_{U}=0$ in this approximation is $D_{\mathrm{cr}}=-(\pi / 2)^{5} \approx$ -9.6 , rather than $D_{\text {cr }}=-8$ as adopted above. We neglect this difference in view of the approximate nature of the solutions and parameter values used.

Estimates of $V_{z}$ and $U_{z}$ are uncertain as they involve complex connections among star formation, the multi-phase structure of the ISM, and the physical mechanisms of launching an outflow. Two plausible estimates given in Appendix B yield consistent results,

$$
\begin{aligned}
R_{U} \simeq & 0.45\left(\frac{U_{z}}{0.3 \mathrm{~km} \mathrm{~s}^{-1}}\right)\left(\frac{h}{0.5 \mathrm{kpc}}\right) \\
& \times\left(\frac{l}{0.1 \mathrm{kpc}}\right)^{-1}\left(\frac{v}{10 \mathrm{~km} \mathrm{~s}^{-1}}\right)^{-1}
\end{aligned}
$$

with $V_{z}$ given by Equation (B2) or Equation (B4).
Equation (14) for the steady-state mean magnetic field can be expressed in terms of directly observable quantities using Equations (7)-(9) for $D$ and $R_{\omega}$, Equation (16) for $D_{\mathrm{cr}}$, and Equation(17) for $R_{U}$. The value of $p_{B}$ can be taken either from observations or from the accompanying estimate (23). For the calculations in Section 4, we use (23), but also tested using the observed values and found the results to be consistent (since the effect of $p_{B}$ on the steady-state magnetic field is weak as long as $\left|p_{B}\right|$ is sufficiently small).

\subsubsection{Dynamo Saturation Mechanism versus Star Formation Rate}

The magnetic field strength established through the magnetic helicity balance is expected to be lower than that arising from magnetostrophic balance: Equation (14) yields a lower value of $\bar{B}$ than Equation (13) provided

$$
\frac{U_{z}}{\Omega h} \lesssim 0.3
$$

For a flat rotation curve $(S=-\Omega), U_{z}=1 \mathrm{~km} \mathrm{~s}^{-1}, \Omega=$ $25 \mathrm{~km} \mathrm{~s}^{-1} \mathrm{kpc}^{-1}$, and $h=0.5 \mathrm{kpc}$, the left-hand side of this inequality is about 0.1 .

\subsection{Magnetic Pitch Angle}

The pitch angle of the mean magnetic field $p_{B}$, is defined as the acute angle between the magnetic field direction and the tangent to the local circumference,

$$
\tan p_{B}=\frac{\bar{B}_{r}}{\bar{B}_{\phi}}
$$

In a trailing spiral, $p_{B}<0$, in contrast to $p_{B}>0$ for a leading one. This is a readily observable quantity that can be used to understand the mechanism that produces the mean magnetic field, as first suggested by Krasheninnikova et al. (1989). In particular, the pitch angle involves the ratio of magnetic field components, and thus depends on fewer galactic parameters than the strength of the mean magnetic field.

\subsubsection{Kinematic Dynamo}

Krasheninnikova et al. (1989) showed that, in the kinematic mean-field dynamo,

$$
\tan p_{B} \simeq-\left(\frac{R_{\alpha}}{\left|R_{\omega}\right|}\right)^{1 / 2} .
$$

Using Equations (4) and (5), we obtain

$$
\tan p_{B} \simeq-\frac{l}{h}\left(\frac{\Omega}{|S|}\right)^{1 / 2} .
$$

We have $\Omega / S=-1$ for a flat rotation curve, and $l$ presumably varies little with the galactocentric radius $r$. Then the magnetic pitch angle varies with $r$ mostly because of the disk flaring, i.e., the increase in $h$ with $r$. In a normalized form, we have

$$
\tan p_{B} \simeq-0.2\left(\frac{\Omega}{|S|}\right)\left(\frac{l}{0.1 \mathrm{kpc}}\right)\left(\frac{h}{0.5 \mathrm{kpc}}\right)^{-1}
$$

\subsubsection{Magnetostrophic Balance}

The value of $p_{B}$ that arises from the magnetostrophic balance can be estimated heuristically by replacing the dynamo 
Table 4

Kendall's Correlation Coefficient $\tau$, the Significance Level $v$ of the Correlation, and the Number of Galaxies (or Galaxy Regions) Involved $N$ for the Inter-dependencies between the Large-scale Magnetic Field Strength Obtained from Observations and from Various Models of Section 3.4

\begin{tabular}{|c|c|c|c|c|c|c|}
\hline \multirow[t]{2}{*}{ Model } & \multicolumn{3}{|c|}{$\bar{B}_{0}^{2}$} & \multicolumn{3}{|c|}{$\tan p_{B}$} \\
\hline & $\tau$ & $v, \%$ & $N$ & $\tau$ & $v, \%$ & $N$ \\
\hline Energy equipartition, Equation (12) & -0.005 & 97 & 20 & & & \\
\hline Kinematic, Equation (20) & & & & 0.013 & 92 & 31 \\
\hline Magnetostrophic balance, Equations (13) and (22) & -0.091 & 57 & 20 & -0.10 & 62 & 15 \\
\hline Magnetic helicity balance, Equations (14) and (23) & -0.405 & 10 & 10 & -0.10 & 62 & 15 \\
\hline
\end{tabular}

Note. None of the correlations pass the 5\% significance level test.

number by its critical value, $R_{\alpha} R_{\omega}=D_{\mathrm{cr}}$. Equation (18) then reduces to

$$
\tan p_{B} \simeq \frac{\left|D_{\mathrm{cr}}\right|^{1 / 2}}{R_{\omega}}
$$

or

$$
\tan p_{B} \simeq-0.15\left(\frac{R_{\omega}}{-19}\right)^{-1}
$$

where $D_{\text {cr }}=-8$.

\subsubsection{Magnetic Helicity Balance}

Under magnetic helicity balance, $\tan p_{B} \simeq\left(R_{U}+\pi^{2}\right) / 4 R_{\omega}=$ $\left(-2 D_{\mathrm{cr}} / \pi\right)^{1 / 2} / R_{\omega} \quad$ (Chamandy et al. 2014), similar to Equation (21) but with $D_{\text {cr }}$ from Equation (16):

$$
\tan p_{B} \simeq-0.13\left(\frac{D_{\mathrm{cr}}}{-10}\right)^{1 / 2}\left(\frac{R_{\omega}}{-19}\right)^{-1} .
$$

In fact, this estimate is independent of the nature of the dynamo nonlinearity as it follows from the steady-state equations for $\bar{B}_{r}$ and $\bar{B}_{\phi}$ alone (see Chamandy et al. 2014 for details) and applies whether or not the galactic outflow is responsible for the dynamo saturation. Equation (23) can readily be expressed in terms of directly observable quantities using Equation (16) for $D_{\text {cr }}$, Equation (17) for $R_{U}$, and Equation (9) for $R_{\omega}$.

\section{TESTING GALACTIC DYNAMO MODELS}

The mean-field galactic dynamo theory, briefly reviewed in Sections 3.3-3.5, predicts specific dependencies of the strength and pitch angle of the mean magnetic field on galactic parameters, which can be tested using observations. Such testing is the subject of this section. Unfortunately, we are not aware of any suitable specific predictions from the primordial or any other alternative theory for the origin of mean magnetic fields.

\subsection{Strength of the Mean Magnetic Field}

For each of the three relationships for the mean magnetic field strength, Equations (12), (13), and (14), we calculated for each galaxy the predicted value of the axisymmetric mean magnetic field strength $\bar{B}_{0}$ averaged over the same area as the observational data. This was only possible for galaxies where all of the data was available for the radial ranges used. All of the dynamo models use the rotation curve; the energyequipartition and magnetostrophic balance models also include the gas density, and the helicity balance model involves the SFR.

To evaluate the quality of each model, we used Pearson's product-moment correlation coefficient, with the expectation that a perfect model would produce a correlation coefficient of unity and poor models would produce low correlation coefficients. Table 4 contains the calculated correlation coefficients between the observed mean magnetic field strengths and the model predictions, with the corresponding scatter plots shown in Figure 2.

The correlation coefficients for all three models are equally low, with high significance levels. Thus, we can only conclude that, with the amount of data at our disposal, none of the three models can be excluded or appears any better than the others to any significant degree.

The magnetic field strength obtained from any dynamo saturation model depends on the ratio $h^{2} / v^{2}$, treated above as a constant. However, unlike the turbulent velocity, $h$ can vary widely between the galaxies and with galactocentric radius within each galaxy. To assess the consequences of treating this ratio as a constant, we varied the value of $h^{2} / v^{2}$, increasing and decreasing it by up to a factor of five for all the galaxies simultaneously, and then repeated the correlation analysis. We found that the correlation coefficients were changed by a minimal amount, and our conclusions regarding all three of the dynamo models remain unaffected.

\subsection{Pitch Angle of the Mean Magnetic Field}

To test theoretical predictions for the pitch angle of the mean magnetic field, Equations (19), (22), and (23), we can use Pearson's linear correlation coefficient as we expect (or hope) to have a simple linear relation between the observed and predicted values. The resulting correlation coefficients are shown in Table 4 and the scatter plots are shown in Figure 3. The predicted pitch angles for a kinematic dynamo and for the magnetostrophic balance depend on the rotation curve alone, and so can be tested for more galaxies than the magnetic helicity balance model, which includes more parameters. The kinematic dynamo, magnetostrophic balance, and helicity balance models all produce weak anti-correlations instead of positive correlations. All three models tend to underestimate the magnitude of the observed pitch angles and both Equation (19) and Equation (23) predict narrower ranges of $p_{B}$ than that observed.

The fact that Equation (19), obtained from a kinematic dynamo model, does not agree with the observed magnetic pitch angles, can just mean that the mean-field dynamos in most, if not all, of the sample galaxies have already entered a non-linear (saturated) stage. The failure of the magnetostrophic balance model to predict the observed pitch angles suggests either that the force balance involved is not the dominant mechanism of the mean-field dynamo saturation or that most or all of the galaxies have a significant outflow across the disk-halo interface. Either way, this implies that $p_{B}$ should depend on the gas density and SFR: both $\Sigma$ and $\Sigma_{*}$ appear in Equation (23) but not in Equations (19) and (22).

The pitch angles predicted by the magnetic helicity balance model are also much smaller than those observed. This is because the actual values of $\left|R_{\omega}\right|$ in Equations (22) and (23) 

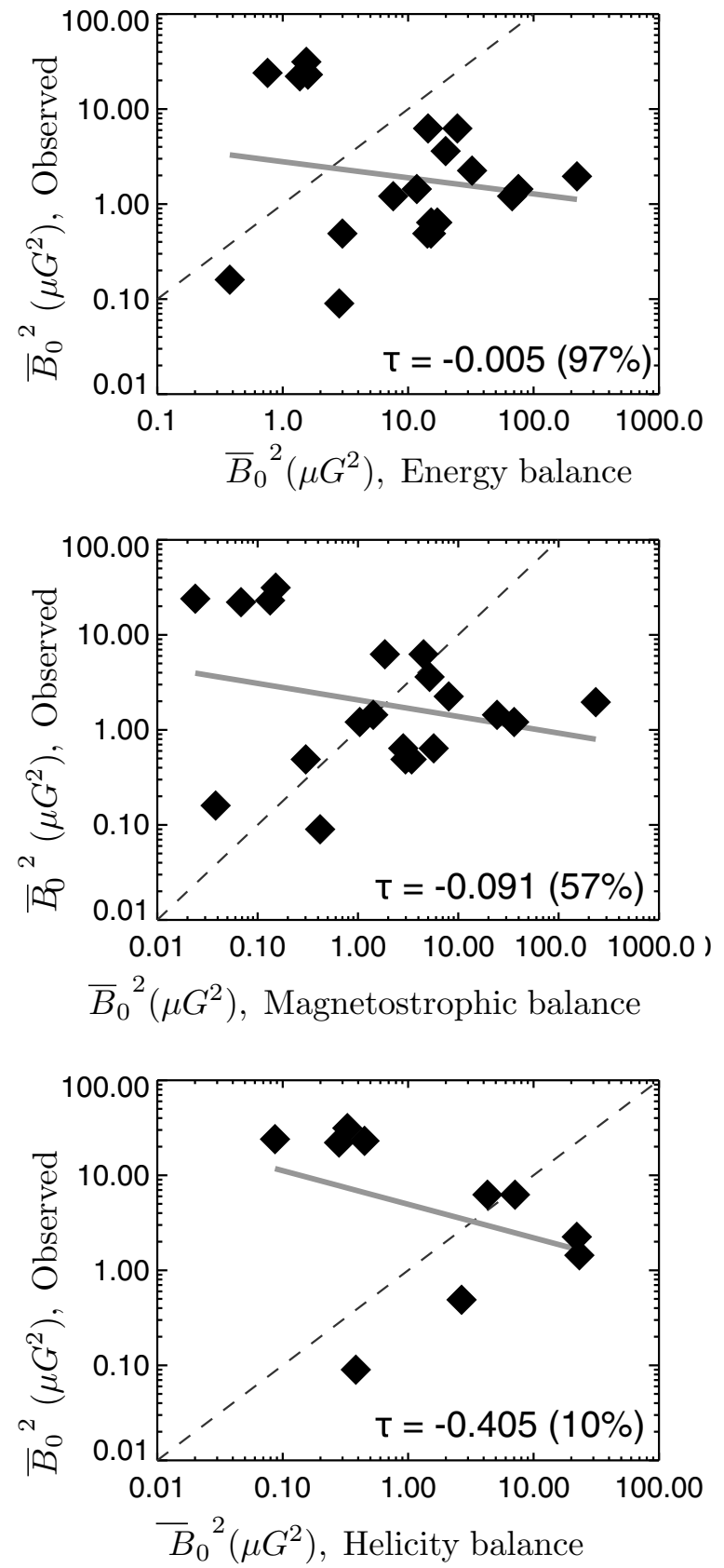

Figure 2. Observed axisymmetric mean magnetic field strength vs. that from various nonlinear dynamo models: energy equipartition with turbulence (top), magnetostrophic balance (middle), and magnetic helicity balance (bottom). The gray lines are the best-fit linear relationship; Pearson's correlation coefficient $r$ and the significance level of the correlation (\%) are shown in each panel.

exceed its normalization value: the strong shear leads to a prediction of a tightly wound up field with small pitch angles. So the dynamo models we have constructed overemphasize the role of shear in determining the mean magnetic field pitch angle.

\section{THE PAIR-WISE CORRELATIONS}

Apart from those for the few nearby galaxies discussed above, the data are very inhomogeneous, especially regarding the diversity of approaches to obtain magnetic field strength. Various authors use either minimum-energy or pressure equipartition estimates to derive the total magnetic field strength from syn-
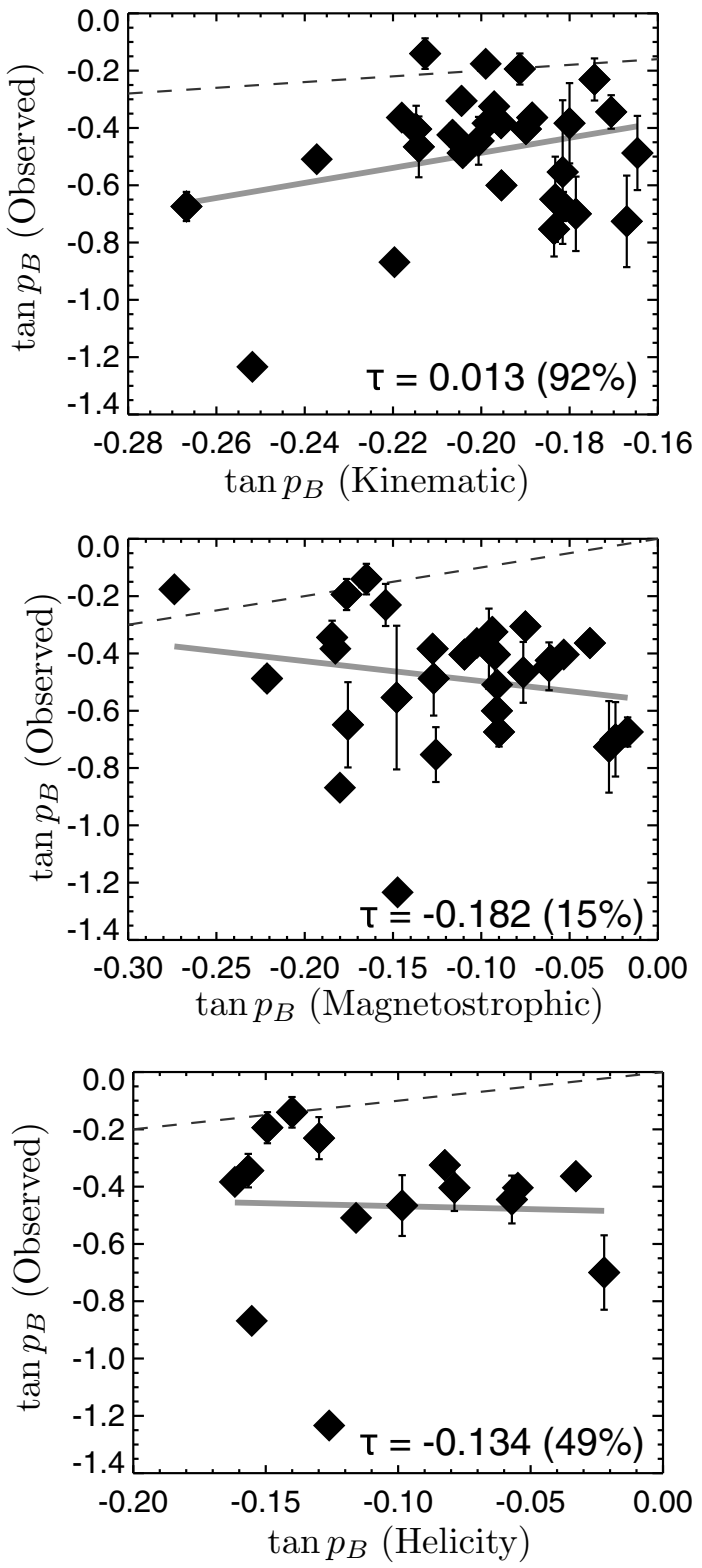

Figure 3. Comparison of the magnetic pitch angles observed and those predicted by the kinematic dynamo (top), nonlinear dynamo with magnetostrophic saturation (middle), and nonlinear dynamo with saturation through helicity balance (bottom). The gray lines are the best-fit linear relationship, while the black line corresponds to the perfect agreement (unit slope).

chrotron intensity. The strength of the mean magnetic field is obtained from the polarized synchrotron intensity, most often without proper regard of depolarization. The estimates are often published without clear indication of the region within the galaxy to which they refer. Moreover, the routinely used assumption of a direct local relation between the cosmic-ray and magnetic energy densities or pressures is likely to be wrong (Stepanov et al. 2014), leading to an overestimated $\bar{B}$ if the linear resolution of the observations is finer than a few hundred parsecs.

The consequences of the undesirable diversity in the existing interpretations of the radio continuum observations of spiral galaxies, and the way to improve the situation, become evident as soon as one attempts to compare the data and develop a coherent picture of magnetic fields in the galaxy sample. Therefore, we present here the pair-wise correlations between 

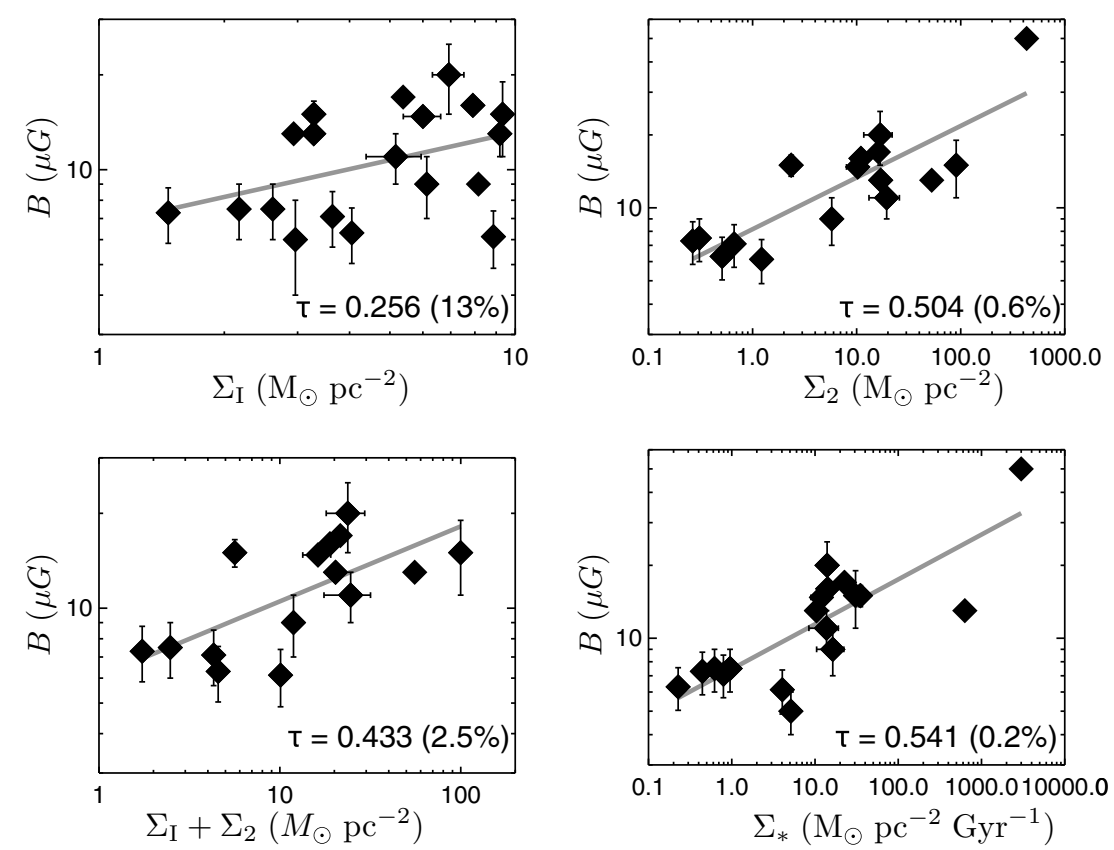

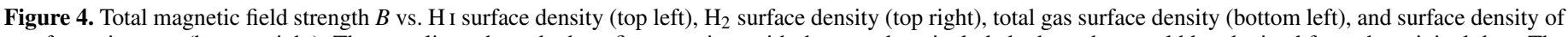

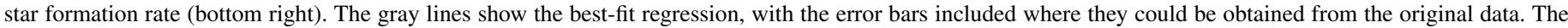
correlation coefficient, $\tau$, and significance level $v$ (in brackets) are given in each panel.

Table 5

Kendall's Correlation Coefficient $\tau$, the Significance level $v$ and the Number of Galaxies (or Galaxy Regions) Involved $N$ in the Pair-wise Correlations of Magnetic Field Parameters and other Characteristics of the Interstellar Medium

\begin{tabular}{|c|c|c|c|c|c|c|c|c|c|c|c|c|}
\hline & \multicolumn{4}{|c|}{$B$} & \multicolumn{4}{|c|}{$\bar{B}$} & \multicolumn{4}{|c|}{$\tan p_{B}$} \\
\hline & $\tau$ & $v, \%$ & $N$ & $k$ & $\tau$ & $v, \%$ & $N$ & $k$ & $\tau$ & $v, \%$ & $N$ & $k$ \\
\hline$B$ & & & & & 0.42 & 1.0 & 20 & $0.76 \pm 0.23$ & & & & \\
\hline$\Sigma_{\mathrm{I}}$ & 0.26 & 13 & 19 & & -0.14 & 36 & 23 & & -0.21 & 10 & 30 & \\
\hline$\Sigma_{2}$ & 0.50 & 0.6 & 16 & $0.21 \pm 0.04$ & 0.02 & 91 & 18 & & -0.36 & 4.3 & 17 & $-0.10 \pm 0.08$ \\
\hline$\Sigma_{\mathrm{I}}+\Sigma_{2}$ & 0.43 & 2.5 & 15 & $0.24 \pm 0.07$ & -0.03 & 85 & 18 & & -0.39 & 2.8 & 17 & $-0.25 \pm 0.13$ \\
\hline$\Sigma_{*}$ & 0.54 & 0.17 & 18 & $0.19 \pm 0.03$ & 0.10 & 52 & 21 & & -0.44 & 2.2 & 15 & $-0.15 \pm 0.09$ \\
\hline$\Omega$ & 0.29 & 6.2 & 22 & & 0.06 & 65 & 26 & & -0.16 & 21 & 31 & \\
\hline$-S$ & 0.34 & 2.7 & 22 & & 0.13 & 34 & 26 & & -0.14 & 26 & 31 & \\
\hline$-\overline{\Omega S}$ & 0.34 & 2.3 & 22 & $0.14 \pm 0.04$ & 0.13 & 34 & 26 & & -0.16 & 22 & 31 & \\
\hline $\bar{B}_{0}$ & & & & & & & & & 0.56 & 0.048 & 20 & $0.51 \pm 0.11$ \\
\hline
\end{tabular}

Notes. Entries shown in bold pass the $v=5 \%$ significance level test. For the significant correlations, we also present the best-fit power-law index $k$ as, e.g., in $B \propto \Sigma_{2}^{0.24 \pm 0.04}$.

the magnetic and other parameters of the galaxies, being aware that their physical significance may be limited.

We calculated Kendall's correlation coefficient $\tau$, given in Table 5, for each pair-wise combination of magnetic field with other ISM parameters. Also shown in the table are the significance levels and the number of galaxies for which we have the required data. We chose to use Kendall's correlation coefficient, instead of the more commonly used Pearson's product-moment correlation coefficient, as Kendall's coefficient is sensitive to nonlinear correlations, and we had no a priori reason to assume that any correlations are linear. The significance level $v$ represents the chance that a correlation could be produced by uncorrelated data; lower significance levels indicate that an observed correlation is more likely to be real. We used the $v=5 \%$ to identify statistically significant correlations. This leads to 10 significant results from the combinations tested.

A summary of the pair-wise correlations is presented in Table 5 and the scatter plots and fits are shown in Figures 4-9.

\subsection{Magnetic Field Strength}

The total magnetic field strength $B$ is significantly correlated with the surface densities of $\mathrm{H}_{2}$ and SFR. However, these variables are themselves correlated through the Schmidt-Kennicutt law, so the two correlations are not independent. The correlation with the SFR is similar to that found by Chyży (2008) and Chyży et al. (2011). The co-variation of $B$ and $\Sigma_{2}$ is consistent with Equation (1) with $k \approx 0.21$, which does not fit any of the standard models such as isotropic two- or one-dimensional compression. Since $k=0.21$ is far below the smallest of the values arising from those models, $k=1 / 2$, the difference cannot be explained by a mixture of various behaviors but rather suggests either that scaling of the magnetic field strength with the gas density alone, Equation (1) fails to capture the relevant physics of the cloud formation, or that Equation (1) is not applicable to data averaged on scales of about $1 \mathrm{kpc}$ (a typical linear resolution of the radio observations used). 

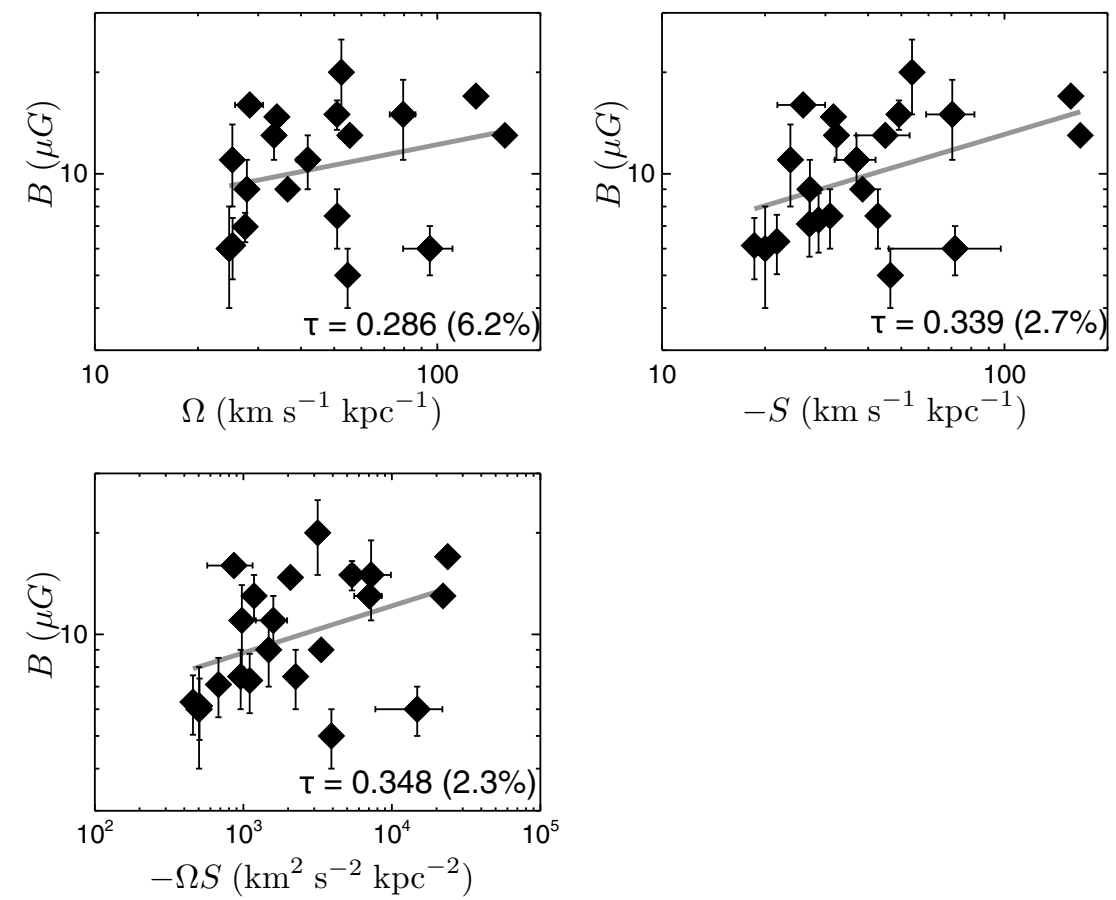

Figure 5. As in Figure 4, but for the total magnetic field strength $B$ vS. angular velocity (top left), rotational shear rate (top right), and their product (bottom left).
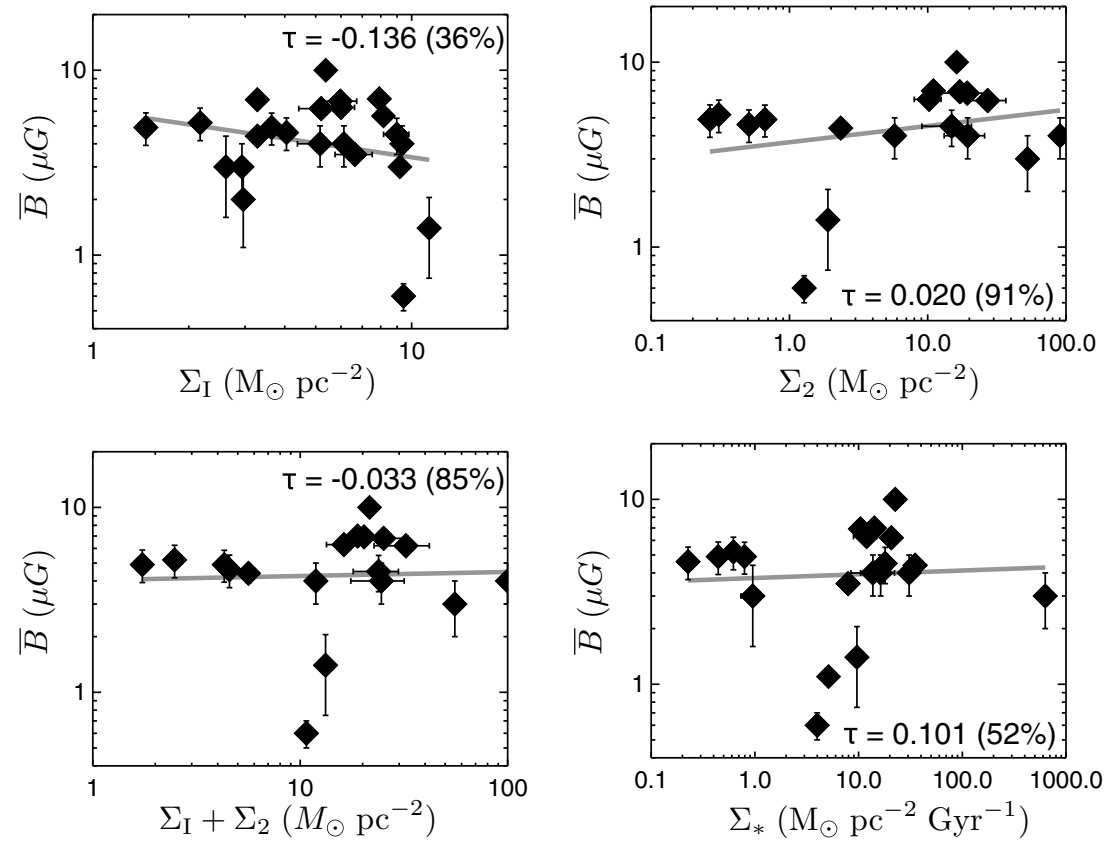

Figure 6. As in Figure 4, but for the mean magnetic field strength $\bar{B}$.

The dependence expected from Equation (2) is recovered if the internal velocity dispersion depends on the cloud density approximately as $v \propto \rho^{-1 / 4}$. This is not inconsistent with Larson's laws (Larson 1981), first obtained for molecular clouds, but then extended to diffuse clouds: $\rho \propto R^{-1.15 \pm 0.15}$ and $v \propto R^{0.4 \pm 0.1}$ (Vazquez-Semadeni et al. 1997), which can be combined to $v \propto \rho^{0.35 \pm 0.10}$. Together with Equation (2), these relations imply $B^{2} \propto \rho^{0.3 \pm 0.1}$, which is consistent within $2 \sigma$ ranges with $B^{2} \propto \rho^{0.5 \pm 0.1}$ obtained here despite the fact that individual clouds are not resolved in the data used.

The strength of the mean magnetic field $\bar{B}$ exhibits no discernible pair-wise correlation with any of the simple variables tested. This is somewhat surprising, especially the lack of correlation with the rotational shear rate $S$ as one can be quite confident that galactic differential rotation does affect the largescale magnetic field. It may be the case that the differential rotation, while necessary for the operation of the dynamo, is not the factor that limits the efficiency of the dynamo and ultimately is not dominant in determining the final magnetic field strength. If so, the correlation between rotation and the field strength may be too weak to be identified at reasonable significance, especially with the limited data sets currently available.

\subsection{Pitch Angle of the Mean Magnetic Field}

The pitch angle of the mean magnetic field is correlated, at better than 5\% significance, with the surface density of molecular hydrogen, the total gas surface density, and the SFR. These three quantities are not independent, as the $\mathrm{H}_{2}$ density is 

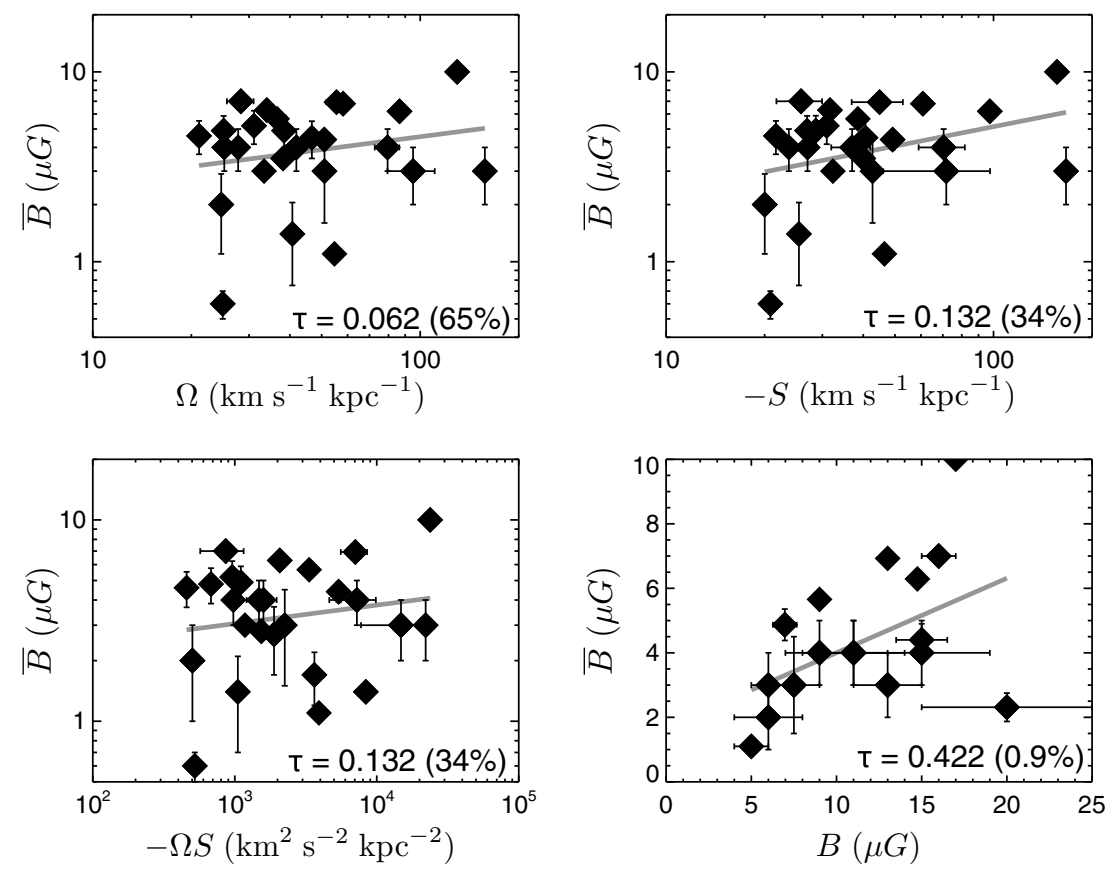

Figure 7. As in Figure 5 but for the mean magnetic field strength $\bar{B}$, including its relation to the total magnetic field strength $B$ (bottom right).
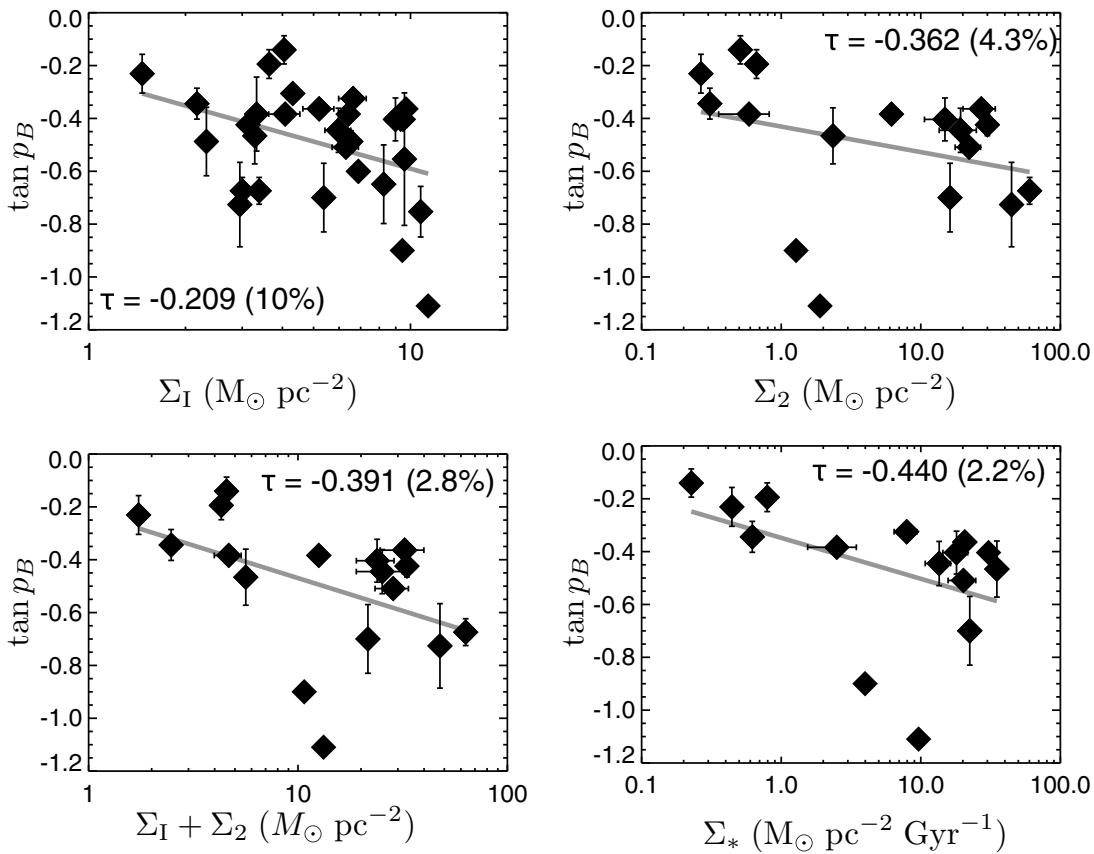

Figure 8. Pitch angle of the mean magnetic field vs. $\Sigma_{\mathrm{I}}$ (top left), $\Sigma_{2}$ (top right), total gas surface density $\Sigma_{\mathrm{I}}+\Sigma_{2}$ (bottom left), and $\Sigma_{*}$ (bottom right). The gray lines show the best-fit regression, with the error bars are included where they could be obtained from the original data. The correlation coefficient, $\tau$, and significance level $v$ are given in each panel.

a substantial part of the total gas density, and the gas density and SFR are related by the Schmidt-Kennicutt law. Of the three variables, $\Sigma_{*}$ exhibits the strongest connection with $p_{B}$, which could imply that the pitch angle is physically related to star formation, whereas the other, weaker correlations reflect relationships between those parameters and the SFR. To clarify this, one would require a more sophisticated statistical analysis and, most importantly, more data.

The tightest and perhaps the most remarkable close correlation that we find is that between the strength of the axisymmetric component of the mean magnetic field $\bar{B}_{0}$ and the magnetic pitch angle. As $\bar{B}_{0}$ gets stronger $p_{B}$ gets smaller: in other words, strong axisymmetric mean fields are more tightly wound than weak ones. Since $\bar{B}_{0}$ has the fastest growth rate of the azimuthal magnetic field patterns according to galactic dynamo theory (Ruzmaikin et al. 1988b) this correlation may provide a way to rank the efficiency of the mean-field dynamos in different galaxies, with smaller $p_{B}$ indicating a more efficient dynamo. However, the interpretation of this correlation should be treated with caution until a predictive link between $\bar{B}_{0}$ and $p_{B}$ is established. Our experience reported in Section 4 suggests that this might be more difficult than one might naively expect. 

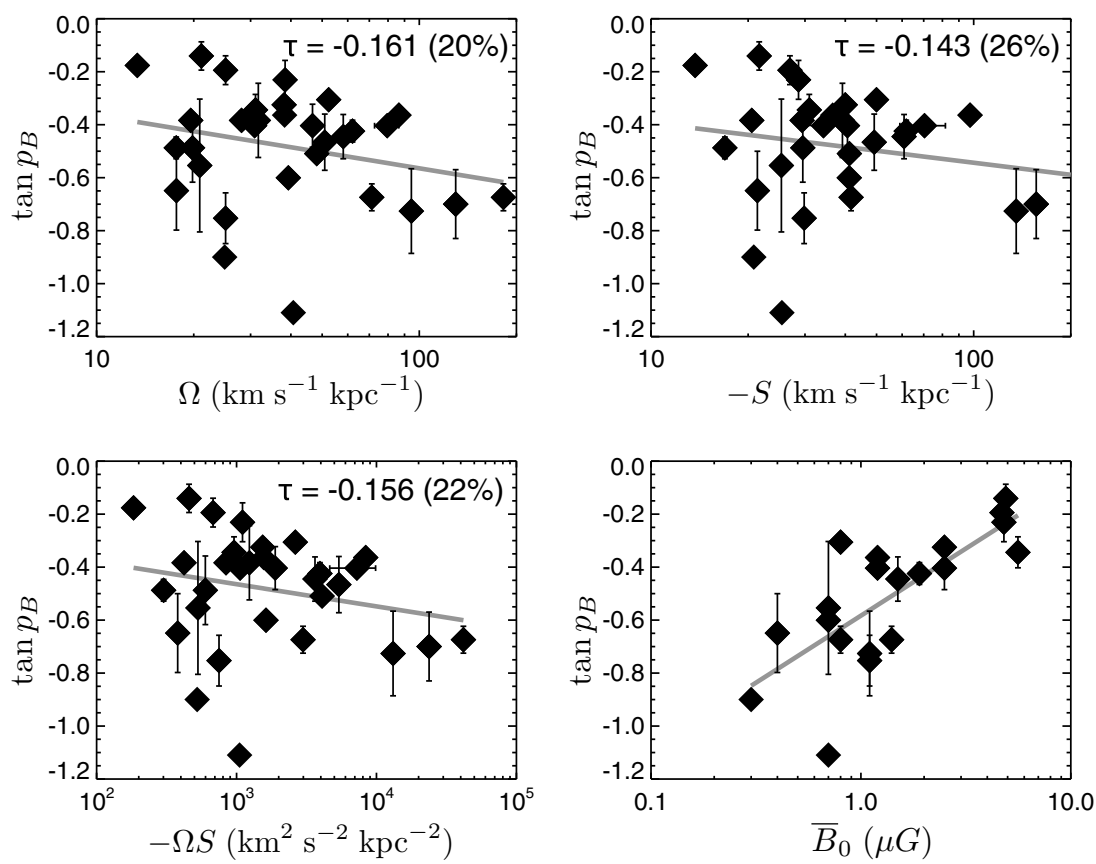

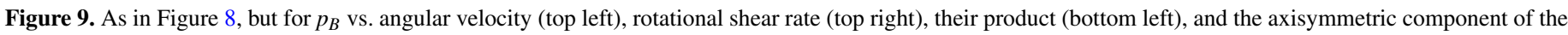
large-scale magnetic field (bottom right).

Perhaps surprisingly, $p_{B}$ shows no significant correlation with the rotational shear $S$ despite the fact that a mean magnetic field must be affected by the galactic differential rotation. We note, however, that the weak negative correlation of $\left|p_{B}\right|$ with $|S|(\Omega$ and $S=r \mathrm{~d} \Omega / \mathrm{d} r$ are functionally related) suggests a decrease in $\left|p_{B}\right|$ as $|S|$ increases, consistent with a tighter winding of magnetic spirals under stronger differential rotation.

As with the strength of the mean magnetic field, a possible explanation of the weak correlation of $p_{B}$ with $S$ is that the pitch angle depends not only on $S$ but also on other parameters such as the SFR, as revealed here. In Section 4 we investigated the correlation of $p_{B}$ with different combinations of parameters derived from specific physical models in Section 3, but this does not improve the correlation. It is more plausible that the magnetic pitch angle is affected by the spiral arms as discussed in Section 3.1. Figure 10 demonstrates a clear correlation between the pitch angles of the spiral arms, $p_{\mathrm{a}}$, and the mean magnetic field, $p_{B}$. Gray crosses show the local pitch angles in M51 obtained by Patrikeev et al. (2006) (their Figure 9); these are the local pitch angles of the total magnetic field and the CO spiral arm segments. The mean value of the difference of the pitch angles is close to zero with the standard deviation of $10^{\circ}$ and the median value of 1.5. The other symbols show the pitch angles of the large-scale magnetic field and spiral arms in several galaxies specified in the figure caption. The mean and median values of the difference between the pitch angles $\left|p_{B}\right|-\left|p_{\mathrm{a}}\right|$ (all data points in Figure 10 except for the local pitch angles in M51) are about $5^{\circ}$ (with the standard deviation of $9^{\circ}$ and only 4 negative values of $\left|p_{B}\right|-\left|p_{\mathrm{a}}\right|$ out of 17 , all from the outer rings in IC 342). The global estimates of $p_{B}$ are plausible to be biased to the interior of spiral arms because of the smaller errors of the polarization angles in the arms where the polarization intensity is generally stronger.

The pitch angles of spiral arms are most often obtained from a global fitting of a logarithmic spiral whereas magnetic pitch angles are obtained in a less restrictive manner and are allowed to vary with radius (and often, with azimuth). This is likely to

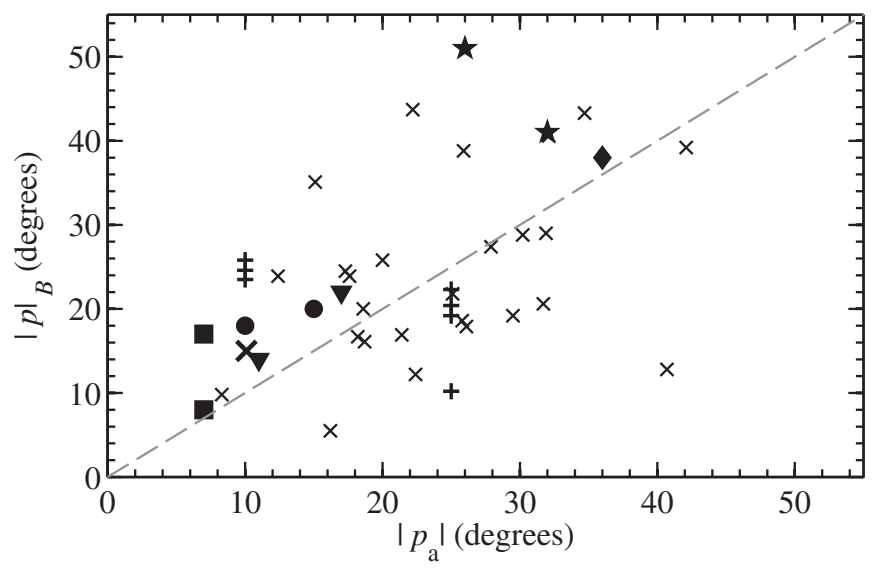

Figure 10. As in Figure 8, but for $p_{B}$ vs. the pitch angle of spiral arms $p_{\mathrm{a}}$ in a selection of galaxies with the data available. M51 (crosses): the local pitch angles of the mean magnetic field and the $\mathrm{CO}$ spiral arms (from Figure 9 in Patrikeev et al. 2006). M33 (stars): $p_{\mathrm{a}}$ from Sandage \& Humphreys (1980, Table 1; measured from the local circumference rather than the local radius as in the original) and $p_{B}$ from the $m=0+2$ fits of Tabatabaei et al. (2008, Table 2), both averaged in the radial ranges $1-3 \mathrm{kpc}$ and $3-5 \mathrm{kpc}$. IC 342 (plus signs): $p_{B}$ from R. Beck (2014, private communication and in preparation), obtained by averaging the pitch angles of the $B$-vectors at $\lambda 6 \mathrm{~cm}$ in sectors of $10^{\circ}$ wide in azimuth and $2 \mathrm{kpc}$ wide in radius in the galaxy plane) and $p_{\mathrm{a}}$ from H I observations of Crosthwaite et al. (2000). M31 (squares): $p_{B}$ from Fletcher et al. (2004), $p_{\mathrm{a}}$ from Nieten et al. (2006). M51 (circles): $p_{B}$ from Fletcher et al. (2011), $p_{\mathrm{a}}$ from Berkhuijsen et al. (1997). M81 (triangles): $p_{B}$ from Krause et al. (1989), $p_{\mathrm{a}}$ from Oort (1974). NGC 6946 (diamond): $p_{B}$ and $p_{\mathrm{a}}$ from Frick et al. (2000). The Orion arm of the Milky Way (large cross): $p_{B}$ from Frick et al. (2001), $p_{\mathrm{a}}$ from Xu et al. (2013). The straight line corresponds to $p_{B}=p_{\mathrm{a}}$.

affect the relation between the pitch angles discussed above. The local magnetic pitch angles in M51 are, on average, very close to those of the $\mathrm{CO}$ spiral arms, but the former are derived from the polarization angles that are known to be affected by anisotropic random fields (Stepanov et al. 2014); hence, the comparison should be treated cautiously as it may not reflect reliably the relation between the local pitch angles of the 
large-scale magnetic field and spiral arms. Despite these caveats, we can conclude that there is a tight correlation between the pitch angles of magnetic field and spiral arms. Nevertheless, they differ systematically, with the integral lines of the largescale magnetic field likely to be more open than the spiral arms, $\left|p_{B}\right|-\left|p_{\mathrm{a}}\right| \simeq 5-10^{\circ}$.

\subsection{Comparison with Earlier Results}

The local relationship between the total magnetic field strength and the SFR in the galaxy NGC 4254 was studied by Chyży (2008) who used 277 locations in the galaxy, all separated by $1.2 \mathrm{kpc}$. He found a strong correlation between the total magnetic field strength and the SFR, $B \propto \Sigma_{*}^{k}$, with Pearson's correlation coefficient of $r=0.93$ and $k=0.18 \pm 0.01$. Our analysis of the global averages for these quantities over many spiral galaxies gave Kendall's correlation coefficient of 0.49 and a significance level of $1.1 \%$ with 15 galaxies, confirming strong correlation (see the bottom right panel of Figure 4). The close agreement between our results and those of Chyży (2008) is more easily seen when we calculate Pearson's correlation coefficient for our data; we obtain $r=0.77$ (at significance level $0.09 \%$ ) for the logarithmic variables, with $k=0.20 \pm 0.05$. Like us, Chyży (2008) found no correlation between the mean magnetic field and the SFR.

Tabatabaei et al. (2013a) find a similar correlation in NGC 6946 with $k=0.14 \pm 0.01$. These authors attribute the difference from Chyży (2008) to the special conditions in NGC 4256 that belongs to the Virgo cluster. However, our results suggest that NGC 6946 may be anomalous in this respect.

Chyży (2008) also analyzed the field regularity $\bar{B} / b$ in NGC 4254 as a function of the local SFR, to find an anticorrelation $\bar{B} / b \propto \Sigma_{*}^{-0.32 \pm 0.01}$ with $r=-0.71$. For the sake of comparison, we also considered $\bar{B} / b$ to obtain $r=-0.44$ (at $13 \%$ significance level) using logarithmic variables. The value of Kendall's correlation coefficient is $\tau=-0.21$ (at $32 \%$ significance, 13 galaxies). The best-fit power law has the form $\bar{B} / b \propto \Sigma_{*}^{-0.15 \pm 0.09}$, significantly shallower than that in NGC 4254.

Tabatabaei et al. (2013a) also find a rather weak anticorrelation of $\bar{B} / b$ and $\Sigma_{*}$ in NGC 6946, with the dependence being nearly flat at $\Sigma_{*} \lesssim 0.01 M_{\odot} \mathrm{yr}^{-1} \mathrm{kpc}^{-2}$ but somewhat steeper at larger SFRs.

It is possible that the difference between these results arises because the relation of $\bar{B} / b$ to $\Sigma_{*}$ varies widely between galaxies in our sample. It is more plausible, however, that it might be caused by the fact that we use the values of $\bar{B}$ and $b$ averaged over relatively large regions in galactic disks (several square kiloparsecs), whereas Chyży used values averaged over the beam area (about $1.5 \mathrm{kpc}^{2}$ ). Then the difference is understandable if $\bar{B} / b$ and/or $\Sigma_{*}$ vary significantly at a scale of the order of a few kiloparsecs. Indeed, from Figure 7(b) of Chyży (2008), $0.2 \lesssim \bar{B} / b \lesssim 1.6$ and $\Sigma_{*}$ varies by a factor of 300 between various locations across NGC 4254.

Chyży et al. (2011) compared magnetic field strengths to the global averages of various galactic parameters for a sample of seven dwarf galaxies. They found a correlation $B \propto \Sigma_{*}^{0.30 \pm 0.04}$ with the correlation coefficient of 0.94 . Heesen et al. (2014) found a similar relation for a sample of 17 galaxies (containing two dwarf galaxies) assuming energy equipartition between cosmic rays and magnetic fields at the $1 \mathrm{kpc}$ scale. This variation is steeper than that obtained here, $B \propto \Sigma_{*}^{0.19 \pm 0.03}$, and by Chyży (2008).
Chyży et al. (2011) also found a correlation coefficient of 0.78 between the total magnetic field and the $\mathrm{H}$ i surface density in their sample of dwarf galaxies, with a power-law exponent of $0.47 \pm 0.09$. Our selection of spiral galaxies showed no correlation of $B$ with the $\mathrm{H}$ I density, but a significant correlation with the density of the molecular gas. Tabatabaei et al. (2013a) also note that the synchrotron intensity in NGC 6946 is only weakly correlated with $\mathrm{H}$ I density, but find a significant correlation with the total gas density, $B \propto\left(\Sigma_{1}+\Sigma_{2}\right)^{m}$ with $m=0.23 \pm 0.01$, evidently dominated by a correlation with molecular gas, $\Sigma_{2}$. Our results are in a good agreement, with $m=0.24 \pm 0.07$.

To conclude, there is a reasonable degree of agreement between the results presented here and earlier work; where there is disagreement this can be plausibly understood as arising from differences in the number and/or type of galaxies in the samples or from differences in the nature of the data used. This gives us some confidence in the results presented here despite the limited size and quality of the data available.

\section{DISCUSSION}

Earlier attempts to establish correlations between galactic magnetic fields and other galactic parameters met with quite limited success. Among a few notable exceptions is the correlation of magnetic field strength in dense interstellar clouds with gas density (e.g., Troland \& Heiles 1986) or, rather, kinetic energy density (Basu 2000). There are claims of a correlation of the orientation of magnetic fields in molecular clouds with that of the magnetic field in the surrounding kiloparsec-wide region (Han \& Zhang 2007; Li \& Henning 2011). If confirmed, such a correlation would be difficult to explain since random magnetic fields at a scale of the order of $100 \mathrm{pc}$, comparable to the size of a region which collapses to form a molecular cloud, are several times stronger than the large-scale magnetic field (see Stepanov et al. 2014, for a review). Thus, any correlation of magnetic field orientations within a cloud and at a scale of the order of a kiloparsec would require a special explanation, which is not immediately obvious.

The general properties of large-scale galactic magnetic fields strongly suggest that they are formed by mean-field dynamo action (Shukurov 2007). However, detailed, quantitative comparisons with the dynamo theory still remain mostly restricted to individual galaxies (especially barred galaxies: Beck et al. 2005; Moss et al. 2007). Here we have made the first steps toward such a comparison in a sample of 20 spiral galaxies. There are numerous caveats to our analysis:

1. The sample of galaxies used is far from being statistically significant, even though it contains all of the galaxies for which the required data have been published in sufficient detail.

2. The sample is very inhomogeneous, with different morphological classes of galaxy and widely varying linear resolution of the radio observations used to estimate their magnetic properties.

3. The observational magnetic field estimates themselves are derived using two different methods (either from energy or pressure equipartition between magnetic fields and cosmic rays or from the Faraday rotation) that involve a range of further assumptions about the ISM properties in each galaxy, often involving presumed (but not confidently established) similarity with the Milky Way. In particular, estimates of magnetic field strength derived from the total and polarized radio emission, when applied at scales less than 
about a kiloparsec, lead to a systematically underestimated random magnetic field and, correspondingly, an overestimated mean magnetic field (Stepanov et al. 2014). Correlations (or anti-correlations) in the spatial distributions of the relativistic and thermal electrons and the interstellar magnetic fields can also significantly affect the magnetic field estimates, which are generally derived assuming that magnetic fluctuations are statistically independent of those in the number density of thermal electrons (Beck et al. 2003). Anisotropy of the random magnetic field produced by the rotational shear and inhomogeneous outflows can affect estimates of the mean magnetic field strength from the degree of polarization (Sokoloff et al. 1998; Beck et al. 2005) and cause discrepancies between estimates obtained from polarized intensity and Faraday rotation (Fletcher et al. 2011; Stepanov et al. 2014).

4. When testing the theoretical predictions for the strength and pitch angle of the large-scale magnetic field, we neglected the variation of the disk thickness with galactocentric radius. However, disk flaring can have a strong affect on the magnetic pitch angle. The correlations discussed in Section 4.2 may become stronger if this factor could be taken into account.

These complications hamper any statistically rigorous testing of the theoretical results with the observational data available. Some of the problems could be resolved in the near future by a careful re-reduction of the existing observations and a systematic use of the existing theoretical results (e.g., Beck et al. 2005); the others require additional observations and theoretical work.

\section{SUMMARY AND CONCLUSIONS}

We have explored correlations between magnetic fields (both random and mean) and the gas density (both atomic and molecular), SFR, angular velocity, and rotational shear in a sample of 20 spiral galaxies. Both pair-wise correlations between the variables and the dependence of the mean magnetic field on parameter complexes suggested by various dynamo models have been tested. The size and statistical quality of the sample are limited, but several correlations are significant and admit physical interpretation.

We found that the total magnetic field strength (dominated by the random magnetic field in most cases) is significantly correlated with the molecular gas density and the SFR, and has a weak or no pair-wise correlations with the atomic gas density and rotational parameters. The correlation with gas density and the lack of correlation with the rotational parameters is not surprising. The relation between gas density and magnetic field strength is shown to be marginally consistent with Larson's relations between the size, density, and velocity dispersion of interstellar gas clouds.

The mean magnetic field strength $\bar{B}$ does not exhibit any significant pair-wise correlation with any of the individual galactic parameters tested, even the rotational shear rate $S$. Correlations of $\bar{B}$ and its pitch angle $p_{B}$ with molecular gas density and star formation are only modest, but still stronger than with $S$. This may appear surprising as there is no obvious direct physical connection between $p_{B}$ and SFR. We interpret this as an indication that the physical connection is between $\bar{B}$ or $p_{B}$ and combinations of the galactic parameters: we turn to the relations predicted by galactic mean-field dynamo theory (other theoretical ideas on the origin and evolution of galactic magnetic fields do not offer testable predictions).

We have found an unexpected close correlation between the strength of the axisymmetric component of the mean magnetic field and the magnetic pitch angle. This would be easily explained if we had found a tight correlation between the shear $S$ and $p_{B}$-strong shear produces efficient mean-field dynamos and preferentially generates azimuthal field over radial field, resulting in a small pitch angle-but we did not. If a well-founded theoretical explanation for this correlation can be uncovered then it may provide an extremely useful diagnostic for the mean fields of galaxies, as the pitch angle is far easier to determine than the strength of the mean magnetic field: it can be directly measured whereas field strength can only be indirectly inferred.

The recent developments in nonlinear galactic dynamos, where the steady state of the large-scale magnetic field is controlled by magnetic helicity balance, indeed predict that both $\bar{B}$ and $p_{B}$ should depend on gas density and the intensity of an outflow from the galactic disk (which can be either a fountain or winds) and, hence, on the SFR.

On the other hand, the pitch angle of the mean magnetic field is correlated with the pitch angle of the spiral arms. We stress that the two pitch angles still differ systematically by about $5^{\circ}$. Such a correlation arises naturally from a one-dimensional compression of magnetic field in the spiral arms.

We have tested a range of predictions of galactic dynamo theory, from the most general ones that only rely on their elementary features, to detailed models based on specific mechanisms for dynamo saturation. The saturation models considered are (1) energy equipartition between the large-scale magnetic field and the turbulent kinetic energy, (2) balance between the Coriolis and Lorentz forces (the magnetostrophic balance), and (3) magnetic helicity balance. In the galaxy sample used, the mean magnetic field strength does not exhibit statistically significant correlations with the parameter combinations corresponding to either of these models. There can be many reasons for the lack of agreement, coming from flaws in both theory and the data inferred from observations. Regarding the theory, not all physical effects, even those relatively well explored, have been included in the models explored. Such effects include radial flows that can affect the magnetic pitch angle (Moss et al. 2000), additional helicity fluxes that can enhance the mean magnetic field in the helicity balance model (Vishniac \& Cho 2001; Vishniac \& Shapovalov 2014), deviations from axial symmetry in the dynamo solutions, etc. Perhaps more importantly, the outflow model of Appendix B may be wrong or oversimplified; more work is required here. In favor of the models we used, they only contain more or less directly observable parameters (unlike the more involved alternatives).

The data used here have been obtained from observations performed with different telescopes, at different frequencies, resolutions and sensitivities. Furthermore, the only unifying feature of the galaxies in the sample is that they are all disk systems; otherwise, they are widely diverse. The problem with the observations can be alleviated with new surveys of representative galaxy samples or by reducing the existing observations to a common set of resolutions, sensitivities, etc.

The importance of this paper is perhaps more the methods used rather than the results. Particularly important aspects of our experience are (1) the need for a physically motivated, simple, and yet realistic nonlinear model of galactic magnetic fields. Such a model ought to be calibrated using the best observational 
data in the Milky Way and the nearest galaxies, and then carefully extended to a larger sample of galaxies. (2) The need for a comprehensive, unbiased, statistically representative database of galactic magnetic fields and of a broad range of relevant galactic properties. The current surge of activity in galactic magnetism connected with the LOFAR and SKA projects offers excellent opportunities in this area.

We are grateful to Rainer Beck for useful comments. and for providing his results on IC 342 prior to publication. Detailed and useful comments of the anonymous referee are gratefully acknowledged. C.V.E. would like to acknowledge the financial support provided by Alberta Innovates. J.C.B. acknowledges support from the Natural Sciences and Engineering Research Council of Canada. A.F. and A.S. have been supported by the Leverhulme Trust (grant RPG-097) and the STFC (grant ST/ L005549/1). We also acknowledge the extensive use of NASA's Astrophysics Data System, and the SIMBAD Astronomical Database operated by the Centre de Données astronomiques de Strasbourg.

\section{APPENDIX A}

\section{CORRECTION FOR UNRESOLVED GRADIENTS IN THE MEAN MAGNETIC FIELD}

Our data set is very diverse covering a wide range of galaxy types and observational parameters. In some galaxies, the resolution was high enough to resolve a significant part of the mean magnetic field, in others, unresolved gradients could have significantly reduced the intensity of polarized emission. In the latter case, a correction for unresolved gradients, relying on a simple model of the large-scale magnetic field $\overline{\boldsymbol{B}}$, can be introduced as follows. In the galaxy sample considered here, this correction was found to be insignificant.

We neglect the vertical component of $\overline{\boldsymbol{B}}$ in comparison with the horizontal ones, $\left|\bar{B}_{z}\right| \ll\left|\bar{B}_{r}\right|,\left|\bar{B}_{\phi}\right|$ in the cylindrical reference frame $(r, \phi, z)$ with the origin at the galactic center and the $z$-axis aligned with the rotation axis. The field strength is assumed to depend on the galactocentric radius and azimuthal angle $\phi$ as

$$
\bar{B}=B_{0} \exp \left(-r / R_{B}\right) \cos \left[m\left(\phi-\beta_{m}\right)\right]
$$

where $m$ is the azimuthal wave number ( $m=0$ corresponds to an axisymmetric field, $m=1$ to a bisymmetric structure, etc.), $\beta_{m}$ is the phase of the $m$ th mode, $R_{B}$ is the radial length scale of the field strength, and $B_{0}$ is the characteristic field strength. As shown by Berkhuijsen et al. (1997; their Appendix A), the Cartesian components $\bar{B}_{x}$ and $\bar{B}_{y}$ of the projection of a horizontal galactic magnetic field in the sky plane (with the $x$-axis aligned with the galaxy's major axis) are given by

$$
\bar{B}_{x}=\bar{B}_{r} \cos \phi-\bar{B}_{\phi} \sin \phi \quad \bar{B}_{y}=\left(\bar{B}_{r} \sin \phi+\bar{B}_{\phi} \cos \phi\right) \cos i
$$

where $i$ is the inclination angle of the galactic disk $(i=0$ corresponds to the face-on view). Using the pitch angle of the magnetic field $p_{B}$, we have

$$
\bar{B}_{r}=\bar{B} \sin p_{B}, \quad \bar{B}_{\phi}=\bar{B} \cos p_{B} .
$$

Tedious but straightforward algebra then leads to

$$
\begin{aligned}
\bar{B}_{\perp}^{2}= & \bar{B}_{x}^{2}+\bar{B}_{y}^{2}=\frac{1}{2} B_{0}^{2} \exp \left(-2 r / R_{B}\right) \\
& \times\left[1+\cos ^{2} i-\sin ^{2} i \cos 2\left(\phi-p_{B}\right)\right] \cos ^{2}\left[m\left(\phi-\beta_{m}\right)\right] .
\end{aligned}
$$

For the sake of simplicity, we only consider the case $m=0$ below.

When observed at a finite resolution, all the variables are averaged across the beam, i.e., over the corresponding ranges of $\phi$ and $r$ in the galaxy plane. The beam shape in the galaxy plane depends on $\phi$ and $i$. If the angular resolution of the observations is $\theta$ and the distance to the galaxy is $D$, the beam width at the minor axis is $2 d / r$ along the azimuth $\phi$ and $2 d / \cos i$ along $r$, where $d=D \tan i$. Assuming that $d \ll r, R_{B}$ and keeping only the leading terms in $d /(r \cos i)$ and $d / R_{B}$, we obtain near the minor axis $(\phi \approx \pi / 2)$ :

$$
\begin{aligned}
\left\langle\bar{B}_{\perp}^{2}\right\rangle & =(4 D \cos i)^{-1} \int_{r-D / \cos i}^{r+D / \cos i} r^{\prime} d r^{\prime} \int_{\phi-D / r}^{\phi+D / r} \bar{B}_{\perp}^{2}\left(r^{\prime}, \phi^{\prime}\right) \mathrm{d} \phi^{\prime} \\
& \approx \frac{1}{2} B_{0}^{2} e^{-2 r / R_{B}}\left[1+\cos ^{2} i-\sin ^{2} i \cos 2\left(\phi-p_{B}\right)\right] \quad(\text { A2 })
\end{aligned}
$$

where $\left\langle\bar{B}_{\perp}^{2}\right\rangle$ is $\bar{B}_{\perp}^{2}$ averaged over the beam area.

Near the major axis, $\phi \approx 0$, the beam size is $2 D$ along $r$ and $2 D / \cos i$ along $\phi$, and Equation (A2) still applies for a narrow beam.

Remarkably, the result is independent of the beam size as long as $d \ll r, R_{B}$ (such a dependence is in the higher-order terms in $d / r$ ), and the main factor which affects the observed field magnitude via unresolved gradients is the galaxy's inclination and azimuthal position within the disk. We have verified that the dependence on $m$ is also insignificant and, for our purposes, we can use $m=0$ without any significant loss of accuracy. Assumptions such as the cosmic-ray-magnetic field equipartition or the statistical independence of thermal and relativistic electron densities and magnetic field, routinely used in deriving the field strength, introduce by far stronger uncertainties.

\section{APPENDIX B}

\section{THE OUTFLOW SPEED}

Both galactic fountains and winds are driven by supernova explosions that produce hot gas. The speed of the hot gas at the base of the outflow averaged over the disk surface, $V_{z}$ depends on the number of stars born per unit time per unit area, roughly estimated as $\Sigma_{*} / M_{*}$, where $M_{*}$ is the average stellar mass. The supernova rate in a galaxy of a radius $R$ is given by

$$
\nu_{\mathrm{SN}}=\delta_{\mathrm{SN}} \pi R^{2} \Sigma_{*} / M_{*}
$$

where $\delta_{\mathrm{SN}} \simeq 8 \times 10^{-3}$ is the fraction of stars that evolve to supernovae (i.e., those in the mass range $10<M / M_{\odot}<40$ ) for the initial mass function of Kroupa (2008), which also has $M_{*}=0.85 M_{\odot}$.

We present two estimates of the galactic outflow speed, one based on energy conservation and the other on a model of the break-out of a superbubble (produced by an OB association) through the galactic layer of neutral gas. The dynamo action constrained by magnetic helicity conservation only requires an outflow through the disk surface, and it is unimportant whether it is a wind or a fountain. In the latter case, the gas returns 
to the disk in the form of dense clouds. As the formation of the clouds would involve intense reconnections, it is likely that the magnetically nontrivial structures are deposited in the halo rather than returned to the dynamo active disk. Thus, our estimates of the outflow speed are independent of the depth of the galactic gravitational potential. The superbubble break-out model of Mac Low \& McCray (1988), used below, includes the acceleration due to gravity.

\section{B.1. Energy Conservation}

The rate of supernova energy supplied per unit area follows as $\delta_{\mathrm{SN}} E_{\mathrm{SN}} \Sigma_{*} / M_{\odot}$, where $E_{\mathrm{SN}} \simeq 10^{51}$ erg is the supernova energy. If a fraction $\eta$ of the supernova energy feeds the outflow, and the fraction of supernovae that occur in OB associations (and thus drive the outflow) is $\epsilon_{\mathrm{SN}}$, the surface density of the energy supply rate to the outflow is

$$
\dot{E} \simeq \epsilon_{\mathrm{SN}} \eta \delta_{\mathrm{SN}} E_{\mathrm{SN}} \Sigma_{*} / M_{*}
$$

and $\epsilon_{\mathrm{SN}} \simeq 0.7$ (Kulkarni \& Heiles 1988). This energy is carried away from the warm gas layer through both faces of the galactic disk at a timescale $h / V_{z}$, so that the energy in the disk is lost at a rate

$$
\dot{E} \simeq 2 \rho_{\mathrm{h}} V_{z}^{3} .
$$

The balance of the energy supply and loss rates in the disk leads to

$$
\begin{aligned}
V_{z} \simeq & \left(\frac{\epsilon_{\mathrm{SN}} \eta \delta_{\mathrm{SN}} E_{\mathrm{SN}} \Sigma_{*}}{2 \rho_{\mathrm{h}} M_{*}}\right)^{1 / 3}=90 \frac{\mathrm{km}}{\mathrm{s}}\left(\frac{\epsilon_{\mathrm{SN}} \eta \delta_{\mathrm{SN}}}{6 \times 10^{-4}}\right)^{1 / 3} \\
& \times\left(\frac{\Sigma_{*}}{1 M_{\odot} \mathrm{pc}^{-2} \mathrm{Gyr}^{-1}}\right)^{1 / 3}\left(\frac{n_{\mathrm{h}}}{10^{-3} \mathrm{~cm}^{-3}}\right)^{-1 / 3} \\
& \times\left(\frac{E_{\mathrm{SN}}}{10^{51} \mathrm{erg}}\right)^{1 / 3}
\end{aligned}
$$

and, from Equation (15),

$$
\begin{aligned}
U_{z} \simeq & 0.3 \frac{\mathrm{km}}{\mathrm{s}}\left(\frac{V_{z}}{90 \mathrm{~km} \mathrm{~s}^{-1}}\right)\left(\frac{f}{0.1}\right)\left(\frac{n_{\mathrm{h}}}{10^{-3} \mathrm{~cm}^{-3}}\right) \\
& \times\left(\frac{h}{0.5 \mathrm{kpc}}\right)^{-1}\left(\frac{\Sigma_{\mathrm{I}}}{1 M_{\odot} \mathrm{pc}^{-2} \mathrm{Gyr}^{-1}}\right)^{-1} .
\end{aligned}
$$

\section{B.2. The Break-out of a Superbubble}

An alternative estimate is based on a model of a hot, expanding superbubble associated with an OB association (Mac Low \& McCray 1988). Using an idealized numerical model and analytical estimates, these authors argue that a superbubble breaks out of the galactic disk when its radius in the plane of about two scale heights of the neutral hydrogen layer. The breakout radius is defines as that where the superbubble expansion starts accelerating. Following Mac Low \& McCray (1988) in treating a superbubble as a large stellar wind bubble, we use the expansion law obtained by Weaver et al. (1977, 1978),

$$
r=\left(\frac{125}{154 \pi}\right)^{1 / 5}\left(\frac{L t^{3}}{\rho}\right)^{1 / 5}
$$

where $r$ is the superbubble radius, $L=\epsilon_{\mathrm{SN}} \eta \nu_{\mathrm{SN}} E_{\mathrm{SN}}$ is the mechanical luminosity provided by the supernovae within an OB association, $t$ is time and $\rho$ is the ambient diffuse gas density. We identify the outflow speed with the superbubble expansion speed when $r=2 h$ to obtain, using the estimate of the supernova rate $(\mathrm{B} 1)$,

$$
\begin{aligned}
V_{z} \simeq & \left.\frac{d r}{d t}\right|_{r=2 h}=\frac{3}{5}\left(\frac{125}{154 \pi}\right)^{1 / 3}\left(\frac{L}{h \Sigma_{\mathrm{I}}}\right)^{1 / 3} \\
= & 100 \frac{\mathrm{km}}{\mathrm{s}}\left(\frac{\epsilon_{\mathrm{SN}} \eta \delta_{\mathrm{SN}}}{6 \times 10^{-4}}\right)^{1 / 3}\left(\frac{\Sigma_{*}}{1 M_{\odot} \mathrm{pc}^{-2} \mathrm{Gyr}^{-1}}\right)^{1 / 3} \\
& \times\left(\frac{\Sigma_{\mathrm{I}}}{1 M_{\odot} \mathrm{pc}^{-2}}\right)^{-1 / 3}\left(\frac{E_{\mathrm{SN}}}{10^{51} \mathrm{erg}}\right)^{1 / 3} \\
& \times\left(\frac{h}{0.5 \mathrm{kpc}}\right)^{-1 / 3}\left(\frac{R}{15 \mathrm{kpc}}\right)^{2 / 3}
\end{aligned}
$$

and then

$$
\begin{aligned}
U_{z} \simeq & 0.3 \frac{\mathrm{km}}{\mathrm{s}}\left(\frac{V_{z}}{100 \mathrm{~km} \mathrm{~s}^{-1}}\right)\left(\frac{f}{0.1}\right)\left(\frac{n_{\mathrm{h}}}{10^{-3} \mathrm{~cm}^{-3}}\right) \\
& \times\left(\frac{h}{0.5 \mathrm{kpc}}\right)^{-1}\left(\frac{\Sigma_{\mathrm{I}}}{1 M_{\odot} \mathrm{pc}^{-2} \mathrm{Gyr}^{-1}}\right)^{-1} .
\end{aligned}
$$

Equations (B2) and (B4) yield practically identical magnitudes of the outflow speed and the same relation to the SFR but involve different, if not unrelated, galactic parameters.

For comparison, Arribas et al. (2014) find that the maximum velocity of ionized gas outflows in a sample of luminous and ultra-luminous infrared galaxies (at low redshifts) scales with the SFR as $V \propto \mathrm{SFR}^{a}$ with $a=0.24 \pm 0.05$ for the SFR derived from infrared luminosity and $a=0.11 \pm 0.04$ for the SFR obtained from an extinction-corrected $\mathrm{H} \alpha$ luminosity. The dependence on the $\mathrm{H} \alpha$-derived star formation density is similar to that on the corresponding SFR, $V \propto \Sigma_{*}^{0.13 \pm 0.03}$. Rupke et al. (2005) find similar results for the outflow speeds of the neutral gas in ultra-luminous infrared galaxies (at redshifts 0-0.5) and four dwarf starburst galaxies, with $a=0.24 \pm 0.04$ for SFR derived from the infrared luminosity. The variation of $V$ with SFR apparently flattens at SFR $\gtrsim 10 M_{\odot} \mathrm{yr}^{-1}$ (Martin 2005; Rupke et al. 2005; Arribas et al. 2014; Martin et al. 2012). The samples of both Arribas et al. (2014) and Rupke et al. (2005) mainly contain such galaxies. Martin (2005) considered galaxies at redshifts $0.042-0.16$, some of which have lower SFRs, to obtain $V \propto \mathrm{SFR}^{0.35 \pm 0.06}$ for the upper envelope of the data points in the ( $V$, SFR) plane (see also Martin et al. 2012). This dependence agrees very well with that in Equations (B2) and (B4), provided SFR $\propto \Sigma_{*}$.

\section{REFERENCES}

Arribas, S., Colina, L., Bellocchi, E., Maiolino, R., \& Villar-Martín, M. 2014, A\&A, 568, A14

Baryshnikova, Yu., Shukurov, A., Ruzmaikin, A., \& Sokoloff, D. D. 1986, A\&A, 177,27

Basu, S. 2000, ApJL, 540, L103

Beck, R. 2007, A\&A, 470, 539

Beck, R. 2012, SSRv, 166, 215

Beck, R., Poezd, A. D., Shukurov, A., \& Sokoloff, D. 1994, A\&A, 289, 94

Beck, R., Brandenburg, A., Moss, D., Shukurov, A., \& Sokoloff, D. 1996, ARA\&A, 34, 155

Beck, R., Shoutenkov, V., Ehle, M., et al. 2002, A\&A, 391, 83

Beck, R., Shukurov, A., Sokoloff, D., \& Wielebinski, R. 2003, A\&A, 411, 99

Beck, R., Fletcher, A., Shukurov, A., et al. 2005, A\&A, 444, 739

Becker, R., Mebold, U., Reif, K., \& van Woerden, H. 1988, A\&A, 203, 21

Berezinskii, V. S., Bulanov, S. V., Dogiel, V. A., \& Ptuskin, V. S. 1990, Astrophysics of Cosmic Rays ed. V. L. Ginzburg (Amsterdam: NorthHolland), 387

Berkhuijsen, E. M., Horellou, C., Krause, M., et al. 1997, A\&A, 318, 700 
Boissier, S., Gil de Paz, A., Boselli, A., et al. 2007, ApJS, 173, 524

Bottema, R., \& Verheijen, M. A. W. 2002, A\&A, 388, 793

Boulares, A., \& Cox, D. P. 1990, ApJ, 365, 544

Brownstein, J. R., \& Moffat, J. W. 2006, ApJ, 636, 721

Calzetti, D., Wu, S.-Y., Hong, S., et al. 2010, ApJ, 714, 1256

Chamandy, L., Shukurov, A., Subramanian, K., \& Stoker, K. 2014, MNRAS, 443, 1867

Chyży, K. T. 2008, A\&A, 482, 755

Chyży, K. T., \& Buta, R. J. 2008, ApJL, 677, L17

Chyży, K. T., Weżgowiec, M., Beck, R., \& Bomans, D. J. 2011, A\&A, 529, A94

Crosthwaite, L. P. 2001, PhD thesis, University of California, Los Angeles

Crosthwaite, L. P., Turner, J. L., \& Ho, P. T. P. 2000, AJ, 119, 1720

de Blok, W. J. G., Walter, F., Brinks, E., et al. 2008, AJ, 136, 2648

Dicaire, I., Carignan, C., Amram, P., et al. 2008, MNRAS, 385, 553

Dumke, M., Krause, M., \& Wielebinski, R. 2000, A\&A, 355, 512

Ehle, M., \& Beck, R. 1993, A\&A, 273, 45

Ehle, M., Beck, R., Haynes, R. F., et al. 1996, A\&A, 306, 73

Fletcher, A., Beck, R., Shukurov, A., Berkhuijsen, E. M., \& Horellou, C. 2011, MNRAS, 412, 2396

Fletcher, A., Berkhuijsen, E. M., Beck, R., \& Shukurov, A. 2004, A\&A, 414, 53

Fletcher, A., \& Shukurov, A. 2001, MNRAS, 325, 312

Frick, P., Beck, R., Shukurov, A., et al. 2000, MNRAS, 318, 925

Frick, P., Stepanov, R., Shukurov, A., \& Sokoloff, D. 2001, MNRAS, 325, 649

Fridman, A. M., Afanasiev, V. L., Dodonov, S. N., et al. 2005, A\&A, 430, 67

Gräeve, R., \& Beck, R. 1988, A\&A, 192, 66

Han, J. L., \& Zhang, J. S. 2007, A\&A, 464, 609

Heald, G. H., Rand, R. J., Benjamin, R. A., Collins, J. A., \& Bland-Hawthorn, J. 2006, ApJ, 636, 181

Heesen, V., Brinks, E., Leroy, A. K., et al. 2014, AJ, 147, 103

Heesen, V., Krause, M., Beck, R., \& Dettmar, R.-J. 2009, A\&A, 506, 1123

Hummel, E., Beck, R., \& Dahlem, M. 1991, A\&A, 248, 23

Iacobelli, M., Haverkorn, M., Orrú, E., et al. 2013, A\&A, 558, A72

Jałocha, J., Bratek, Ł., Kutschera, M., \& Skindzier, P. 2010, MNRAS, 406, 2805

Kahn, F. D., \& Brett, L. 1993, MNRAS, 263, 37

Kennicutt, R. C. 1989, ApJ, 344, 685

Kennicutt, R. C. 1998, ApJ, 498, 541

Kleeorin, N., Moss, D., Rogachevskii, I., \& Sokoloff, D. 2000, A\&A, 361, L5

Kleeorin, N., Moss, D., Rogachevskii, I., \& Sokoloff, D. 2002, A\&A, 387, 453

Kleeorin, N., Moss, D., Rogachevskii, I., \& Sokoloff, D. 2003, A\&A, 400, 9

Klein, U., Wielebinski, R., \& Morsi, H. W. 1988, A\&A, 190, 41

Krasheninnikova, Y, Ruzmaikin, A., Sokoloff, D., \& Shukurov, A. 1989, A\&A, 213,19

Krause, M., Beck, R., \& Hummel, E. 1989, A\&A, 217, 17

Krause, M., Wielebinski, R., \& Dumke, M. 2006, A\&A, 448, 133

Kroupa, P. 2008, in ASP Conf. Ser. 390, Pathways through an Eclectic Universe, ed. J. H. Knapen, T. J. Mahoney, \& A. Vazdekis (San Francisco, CA: ASP), 3

Kulkarni, S. R., \& Heiles, C. 1988, in Galactic and Extragalactic Radio Astronomy (2nd ed.), ed. K. I. Kellermann \& G. L. Verschuur (Berlin: Springer), 95

Kulsrud, R. M. 1999, ARA\&A, 37, 37

Kulsrud, R. M., \& Zweibel, E. G. 2008, RPPh, 71, 046901

Larson, R. B. 1981, MNRAS, 194, 809

Leroy, A. K., Walter, F., Brinks, E., et al. 2008, AJ, 136, 2782

Li, H.-B., \& Henning, T. 2011, Natur, 479, 499

Lo, K. Y., Cheung, K. W., Masson, C. R., et al. 1987, ApJ, 312, 574

Mac Low, M.-M., \& McCray, R. 1988, ApJ, 324, 776

Martin, C. L. 2005, ApJ, 621, 227

Martin, C. L., Shapley, A. E., Coil, A. L., et al. 2012, ApJ, 760, 127

Mestel, L., \& Paris, R. B. 1984, A\&A, 136, 98

Misiriotis, A., Popescu, C. C., Tuffs, R., \& Kylafis, N. D. 2001, A\&A, 372, 775
Moss, D., Shukurov, A., \& Sokoloff, D. 2000, A\&A, 358, 1142

Moss, D., Shukurov, A., Sokoloff, D. D., Beck, R., \& Fletcher, A. 2001, A\&A, 380,55

Moss, D., Shukurov, A., Sokoloff, D. D., Berkhuijsen, E. M., \& Beck, R. 1998, A\&A, 335, 500

Moss, D., Snodin, A. P., Englmaier, P., et al. 2007, A\&A, 465, 157

Nieten, C., Neininger, N., Guélin, M., et al. 2006, A\&A, 453, 459

Norman, C. A., \& Ikeuchi, S. 1989, ApJ, 345, 372

Oort, J. H. 1974, in IAU Symp. 58, The Formation and Dynamics of Galaxies, ed. J. R. D. Shakeshaft (Dordrecht: Reidel), 375

Patrikeev, I., Fletcher, A., Stepanov, R., et al. 2006, A\&A, 458, 441

Rahman, N., Bolatto, A. D., Wong, T., et al. 2011, ApJ, 730, 72

Rohde, R., Beck, R., \& Elstner, D. 1999, A\&A, 350, 423

Rupke, D. S., Veilleux, S., \& Sanders, D. B. 2005, ApJS, 160, 115

Ruzmaikin, A., Sokoloff, D., \& Shukurov, A. 1985, A\&A, 148, 335

Ruzmaikin, A., Sokoloff, D., \& Shukurov, A. 1988a, Natur, 336, 341

Ruzmaikin, A. A., \& Shukurov, A. M. 1981, SvA, 25, 553

Ruzmaikin, A. A., Shukurov, A. M., \& Sokoloff, D. D. 1988b, Magnetic Fields of Galaxies (Dordrecht: Kluwer)

Sandage, A., \& Humphreys, R. M. 1980, ApJ, 236, L1 (erratum 244, L35 [1981])

Shukurov, A. 2007, in Mathematical Aspects of Natural Dynamos, ed. E. Dormy \& A. M. Soward (London: Chapman \& Hall/CRC), 313

Shukurov, A., \& Sokoloff, D. 2008, in Les Houches, Session LXXXVIII, 2007, Dynamos, ed. Ph. Cardin \& L. F. Cugliandolo (Amsterdam: Elsevier), 251

Shukurov, A., Sokoloff, D., Subramanian, K., \& Brandenburg, A. 2006, A\&A, 448, L33

Sofue, Y., Tutui, Y., Honma, M., et al. 1999, ApJ, 523, 136

Soida, M., Beck, R., Urbanik, M., \& Braine, J. 2002, A\&A, 394, 47

Soida, M., Krause, M., Dettmar, R.-J., \& Urbanik, M. 2011, A\&A, 531, A127

Soida, M., Urbanik, M., Beck, R., Wielebinski, R., \& Balkowski, C. 2001, A\&A, 378,40

Sokoloff, D. D., Bykov, A. A., Shukurov, A., et al. 1998, MNRAS, 299, 189 (erratum 303, 207)

Sorai, K., Nakai, N., Kuno, N., Nishiyama, K., \& Hasegawa, T. 2000, PASJ, 52,785

Starchenko, S. V., \& Shukurov, A. M. 1989, A\&A, 214, 47

Stepanov, R., Shukurov, A., Fletcher, A., et al. 2014, MNRAS, 437, 2201

Sur, S., Shukurov, A., \& Subramanian, K. 2007, MNRAS, 377, 874

Tabatabaei, F. S., \& Berkhuijsen, E. M. 2010, A\&A, 517, A77

Tabatabaei, F. S., Berkhuijsen, E. M., Frick, P., Beck, R., \& Schinnerer, E. 2013a, A\&A, 557, A129

Tabatabaei, F. S., Krause, M., Fletcher, A., \& Beck, R. 2008, A\&A, 490, 1005

Tabatabaei, F. S., Schinnerer, E., Murphy, E. J., et al. 2013b, A\&A, 552, A19

Tempel, E., \& Tenjes, P. 2006, MNRAS, 371, 1269

Thornley, M. D., \& Mundy, L. G. 1997, ApJ, 490, 682

Troland, T. H., \& Heiles, C. 1986, ApJ, 301, 339

Vazquez-Semadeni, E., Ballesteros-Paredes, J., \& Rodriguez, L. F. 1997, ApJ, 474, 292

Verley, S., Corbelli, E., Giovanardi, C., \& Hunt, L. K. 2009, A\&A, 493, 453

Vishniac, E. T., \& Cho, J. 2001, ApJ, 500, 752

Vishniac, E. T., \& Shapovalov, D. 2014, ApJ, 780, 144

Waller, W. H., Kleinmann, S. G., \& Ricker, G. R. 1988, AJ, 95, 1057

Warmels, R. H. 1988, A\&AS, 72, 427

Weaver, R., McCray, R., Castor, J., Shapiro, P., \& Moore, R. 1977, ApJ, 218,377

Weaver, R., McCray, R., Castor, J., Shapiro, P., \& Moore, R. 1978, ApJ, 220,742

Wong, T., \& Blitz, L. 2002, ApJ, 569, 157

Xu, Y., Li, J. J., Reid, M. J., et al. 2013, ApJ, 769, 15

Yim, K., Wong, T., Howk, J. C., \& van der Hulst, J. M. 2011, AJ, 141, 48

Zweibel, E. G, \& Heiles, C. 1997, Natur, 385, 131 